\title{
On Expansion and Contraction of DL-Lite Knowledge Bases (Technical Report)
}

\author{
Dmitriy Zheleznyakov ${ }^{\mathrm{a}}$, Evgeny Kharlamov ${ }^{\mathrm{b}}$, Werner Nutt $^{\mathrm{c}}$, Diego Calvanese ${ }^{\mathrm{c}}$ \\ ${ }^{a}$ Ocado Technology, Hatfield AL10 9UL, United Kingdom. \\ ${ }^{b}$ University of Oxford, Department of Computer Science, Wolfson Building, Parks Road, OX1 3QD, Oxford, UK \\ ${ }^{c}$ Free University of Bozen-Bolzano, Faculty of Computer Science, Piazza Domenicani 3, 39100 Bolzano, Italy.
}

\begin{abstract}
Knowledge bases (KBs) are not static entities: new information constantly appears and some of the previous knowledge becomes obsolete. In order to reflect this evolution of knowledge, KBs should be expanded with the new knowledge and contracted from the obsolete one. This problem is well-studied for propositional but much less for first-order KBs. In this work we investigate knowledge expansion and contraction for KBs expressed in DL-Lite, a family of description logics (DLs) that underlie the tractable fragment OWL 2 QL of the Web Ontology Language OWL 2. We start with a novel knowledge evolution framework and natural postulates that evolution should respect, and compare our postulates to the well-established AGM postulates. We then review well-known model and formula-based approaches for expansion and contraction for propositional theories and show how they can be adapted to the case of DL-Lite. In particular, we show intrinsic limitations of model-based approaches: besides the fact that some of them do not respect the postulates we have established, they ignore the structural properties of KBs. This leads to undesired properties of evolution results: evolution of DL-Lite KBs cannot be captured in DL-Lite. Moreover, we show that well-known formula-based approaches are also not appropriate for DL-Lite expansion and contraction: they either have a high complexity of computation, or they produce logical theories that cannot be expressed in DL-Lite. Thus, we propose a novel formula-based approach that respects our principles and for which evolution is expressible in DL-Lite. For this approach we also propose polynomial time deterministic algorithms to compute evolution of $D L$-Lite KBs when evolution affects only factual data.
\end{abstract}

Keywords: Knowledge Evolution, Knowledge Expansion, Knowledge Contraction, DL-Lite, Semantics, Complexity, Algorithms.

\section{Introduction}

Description Logics (DLs) provide excellent mechanisms for representing structured knowledge as knowledge bases (KBs), and as such they constitute the foundations for the various variants of OWL 2, the standard ontology language of the Semantic $\mathrm{Web}{ }^{1} \mathrm{KBs}$ have traditionally been used for modeling at the intensional level the static and structural aspects of application domains [1]. Recently, however, the scope of KBs has broadened, and they are now used also for providing support in the maintenance and evolution phase of information systems [2]. Moreover, KBs are considered to be the premium mechanism through which services operating in a Web context can be accessed, both by human users and by other services [3, 4, 5]. Supporting all these activities, makes it necessary to equip DL systems with additional kinds of inference servicies that go beyond the traditional ones of satisfiability, subsumption, and query answering provided by current DL inference engines. A critical one, and the subject of this paper, is that of $K B$ evolution [6].

Email addresses: d. zheleznyakov@ocado. com (Dmitriy Zheleznyakov), evgeny. kharlamov@cs .ox.ac.uk (Evgeny Kharlamov),

Werner.Nutt@unibz.it (Werner Nutt), Diego.Calvanese@unibz.it (Diego Calvanese)

1http://www.w3.org/TR/owl2-overview/
In $\mathrm{KB}$ evolution the task is to incorporate new knowledge $\mathcal{N}$ into an existing $\mathrm{KB} \mathcal{K}$, or to delete some obsolete knowledge $\mathcal{N}$ from $\mathcal{K}$, in order to take into account changes that occur in the underlying domain of interest [7]. The former evolution task is typically referred to as knowledge expansion and the latter as contraction. In general, the new (resp., obsolete) knowledge is represented by a set of formulas denoting those properties that should be true (resp., false) after the ontology has evolved. In the case where the new knowledge interacts in an undesirable way with the knowledge in the ontology, e.g., by causing the ontology or relevant parts of it to become unsatisfiable, the new knowledge cannot simply be added to the ontology. Instead, suitable changes need to be made in the ontology so as to avoid the undesirable interaction, e.g., by deleting parts of the ontology that conflict with the new knowledge. Different choices are possible, corresponding to different semantics for knowledge evolution [7, 8, 9, 10, 11, 12].

The main two types of semantics that were proposed for the case of propositional knowledge are model-based [9] and formula-based [10]. In model-based semantics the idea is to resolve the undesirable interaction at the level of models of $\mathcal{K}$ and $\mathcal{N}$. For example, in model-based expansion the result of evolution are those models of $\mathcal{N}$ that are minimally distant from the ones of $\mathcal{K}$, where a suitable notion of distance needs to be chosen, possibly depending on the application. In formula- 
based semantics the idea is to do evolution at the level of the deductive closure of the formulae from $\mathcal{K}$ and $\mathcal{N}$. Since many (possibly counter-intuitive) semantics can be defined within the model or formula-based paradigm, a number of evolution postulates [7, 10] have been proposed and they define natural properties a semantics should respect. It is thus common to verify for each evolution semantics whether it satisfies the postulates.

For the case of propositional knowledge, there is a thorough understanding of semantics as well as of computational properties of both expansion and contraction. The situation is however much less clear when it comes to DL KBs, which are decidable first-order logic theories. Differently from the propositional case, in general they admit infinite sets of models and infinite deductive closures. Moreover, going from propositional letters to first-order predicates and interpretations, on the one hand calls for novel postulates underlying the semantics of evolution, and on the other hand broadens the spectrum of possibilities for defining such semantics. A number of attempts have been made to adapt approaches for the evolution of propositional knowledge to the case of DLs, cf. [11, 12, 13, 14] (see also the detailed discussion of related work in Section 7). However, there is no thorough understanding of evolution from the foundational point of view even for DLs with the most favorable computational properties, such as the logics of the DL-Lite [15] and $\mathcal{E} \mathcal{L}$ [16] families, which are at the basis of two tractable fragments of OWL 2.

In this work we address this problem and propose an exhaustive study of evolution for DL-Lite. In particular, we address the problem considering three dimensions:

1. knowledge evolution tasks: we study how knowledge can be expanded or contracted;

2. type of evolution semantics: we study model-based and formula-based semantics;

3. evolution granularity: we study when evolution affects the TBox (for terminological knowledge), or the ABox (for assertional knowledge), or both of them.

We provide the following contributions:

- We propose a knowledge expansion and contraction framework that accounts for TBox, ABox, and general KB evolution (Section 3.1).

- We propose natural evolution postulates and show how they are related to the well-known AGM postulates (Sections 3.2 and 3.3 .

- We show how one can rigorously extend propositional model-based evolution semantics to the first-order case, defining a five-dimensional space ${ }^{2}$ of possible options, comprising $3 \cdot 2^{4}$ model-based evolution semantics for DLs

\footnotetext{
${ }^{2}$ The dimensions are (see Section 4 for details): (1) ABox vs. TBox vs. general evolution; (2) expansion vs. contraction; (3) global vs. local; (4) symbol vs. atom; (5) set inclusion vs. cardinality.
}

that essentially include all previously proposed modelbased approaches for DLs (Section 4) 3 For most of these semantics and the case of $D L$-Lite KBs we prove negative expressibility results: in general evolution of $D L$-Lite KBs cannot be expressed as a DL-Lite KB.

- We investigate formula-based evolution for DL-Lite. In particular, for known formula-based evolution approaches [10] we show intractability of computing evolution results for DL-Lite KBs. Moreover, we propose a practical though non-deterministic approach for general KB evolution, which turns out to become deterministic for ABox evolution; for both cases we develop practical algorithms (Section 4).

Delta from Previous Publications. This article is based on our conference publication [17] while it significantly extends it in several important directions. First, [17] only considered knowledge expansion, and thus all results on contraction in the current article are new. Second, in [17] we had negative evolution results for only a few out of the $3 \cdot 2^{3}$ knowledge expansion semantics, while here we show negative results for all but three of them. Third, we strengthen the coNP-hardness results for the WIDTIO formula-based semantics presented in [17]. Fourth, in contrast to the current article, [17] did not discuss how AGM postulates as well as various formula-based semantics are related to our postulates. Finally, due to limited space, we did not include in [17] most of the proofs, while in this article we included many more proofs, details, and explanations.

Structure of the Paper. In Section 2, we review the definition of DL-Lite. In Section 3 , we present our evolution framework, including a comparison of our and the AGM postulates. Then, in Section 4, we generalize model-based semantics for the evolution of propositional KBs to the first-order level and investigate whether these semantics can be captured with the expressive means of DL-Lite. In Section 5, we apply formula-based approaches to DL-Lite evolution and study their computational properties. In Section 6, we show that for ABox evolution all formula-based semantics coincide for DL-Lite and that this task is computationally feasible. In Section 7 , we discuss related work, and in Section 8 we conclude the article and discuss future work.

\section{Preliminaries}

We now introduce some notions of Description Logics (DLs) that are needed for understanding the concepts used in this paper; more details can be found in [18].

A DL knowledge base $(\mathrm{KB}) \mathcal{K}=\mathcal{T} \cup \mathcal{A}$ is the union of two sets of assertions (or axioms), those representing the intensional-level of the $\mathrm{KB}$, that is, the general knowledge, and constituting the $T B o x \mathcal{T}$, and those providing information on the instance-level of the $\mathrm{KB}$, and constituting the $A B$ ox $\mathcal{A}$.

\footnotetext{
${ }^{3}$ Note that our proposal for model-based semantics works for any description logic and is not specific for $D L$-Lite.
} 
In our work we consider the DL-Lite family [15, 19] of DLs, which is at the basis of the tractable fragment OWL 2 QL [20] of OWL 2 [21, 22].

All logics of the $D L$-Lite family allow for constructing $b a$ sic concepts $B$, (complex) concepts $C$, and (complex) roles $R$ according to the following grammar:

$$
B::=A|\exists R, \quad C::=B| \neg B, \quad R::=P \mid P^{-} .
$$

where $A$ denotes an atomic concept and $P$ an atomic role, which are just names.

A DL-Lite core $_{\text {TBox }}$ consists of concept inclusion assertions of the form

$$
B \sqsubseteq C .
$$

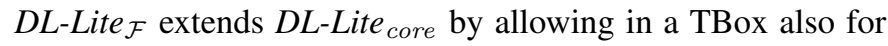
functionality assertions of the form

$$
\text { (funct } R \text { ). }
$$

$D L$-Lite $_{\mathcal{F} \mathcal{R}}$ allows in addition for role inclusion assertions of the form

$$
R_{1} \sqsubseteq R_{2},
$$

in such a way that if $R_{1} \sqsubseteq P_{2}$ or $R_{1} \sqsubseteq P_{2}^{-}$appears in a TBox $\mathcal{T}$, then neither (funct $P_{2}$ ) nor (funct $P_{2}^{-}$) appears in $\mathcal{T}$. This syntactic restriction is necessary to keep reasoning in the logic tractable [19].

ABoxes in DL-Lite core $_{\text {e }}, D L$-Lite F $_{\mathcal{F}}$, and DL-Lite FR $_{\mathcal{R}}$ consist of membership assertions of the form

$$
B(a) \quad \text { or } \quad P(a, b),
$$

where $a$ and $b$ denote constants.

In the following, when we write DL-Lite without a subscript, we mean any of the three logics introduced above.

The semantics of DL-Lite KBs is given in the standard way, using first order interpretations, all over the same infinite countable domain $\Delta$. An interpretation $\mathcal{I}$ is a (partial) function $\mathcal{I}$ that assigns to each concept $C$ a subset $C^{\mathcal{I}}$ of $\Delta$, and to each role $R$ a binary relation $R^{\mathcal{I}}$ over $\Delta$ in such a way that

$$
\begin{aligned}
& \left(P^{-}\right)^{\mathcal{I}}=\left\{(b, a) \mid(a, b) \in P^{\mathcal{I}}\right\}, \\
& (\exists R)^{\mathcal{I}}=\left\{a \mid \exists b \cdot(a, b) \in R^{\mathcal{I}}\right\}, \\
& (\neg B)^{\mathcal{I}}=\Delta \backslash B^{\mathcal{I}} .
\end{aligned}
$$

We assume that $\Delta$ contains the constants and that $a^{\mathcal{I}}=a$, for each constant $a$, i.e., we adopt standard names. Alternatively, we view an interpretation as a set of atoms and say that $A(a) \in \mathcal{I}$ iff $a \in A^{\mathcal{I}}$, and that $P(a, b) \in \mathcal{I}$ iff $(a, b) \in P^{\mathcal{I}}$. An interpretation $\mathcal{I}$ is a model of a membership assertion $B(a)$ if $a \in B^{\mathcal{I}}$ and of $P(a, b)$ if $(a, b) \in P^{\mathcal{I}}$, of an inclusion assertion $E_{1} \sqsubseteq E_{2}$ if $E_{1}^{\mathcal{I}} \subseteq E_{2}^{\mathcal{I}}$, and of a functionality assertion (funct $R$ ) if the relation $R^{\mathcal{I}}$ is a function, that is, for all $a, a_{1}, a_{2} \in \Delta$ we have that $\left\{\left(a, a_{1}\right),\left(a, a_{2}\right)\right\} \subseteq R^{\mathcal{I}}$ implies $a_{1}=a_{2}$.

As usual, we write $\mathcal{I} \models \alpha$ if $\mathcal{I}$ is a model of an assertion $\alpha$, and $\mathcal{I} \models \mathcal{K}$ if $\mathcal{I} \models \alpha$ for each assertion $\alpha$ in $\mathcal{K}$. We use $\operatorname{Mod}(\mathcal{K})$ to denote the set of all models of $\mathcal{K}$. A KB is satisfiable if it has at least one model and it is coheren $4^{4}$ if for every atomic concept and atomic role $S$ occurring in $\mathcal{K}$ there is an $\mathcal{I} \in \operatorname{Mod}(\mathcal{K})$ such that $S^{\mathcal{I}} \neq \emptyset$. We use entailment, $\mathcal{K} \models \mathcal{K}^{\prime}$, and equivalence, $\mathcal{K} \equiv \mathcal{K}^{\prime}$, on KBs in the standard sense. Given a TBox $\mathcal{T}$, we say that an $\mathrm{ABox} \mathcal{A} \mathcal{T}$-entails an $\mathrm{ABox} \mathcal{A}^{\prime}$, denoted $\mathcal{A} \models \mathcal{T} \mathcal{A}^{\prime}$, if $\mathcal{T} \cup \mathcal{A} \models \mathcal{A}^{\prime}$, and that $\mathcal{A}$ is $\mathcal{T}$-equivalent to $\mathcal{A}^{\prime}$, denoted $\mathcal{A} \equiv \mathcal{T} \mathcal{A}^{\prime}$, if $\mathcal{A} \models_{\mathcal{T}} \mathcal{A}^{\prime}$ and $\mathcal{A}^{\prime} \models_{\mathcal{T}} \mathcal{A}$. The deductive closure of a TBox $\mathcal{T}$, denoted $\operatorname{cl}(\mathcal{T})$, is the set of all TBox assertions $\alpha$ such that $\mathcal{T} \models \alpha$. Similarly, the deductive closure of an ABox $\mathcal{A}$ (w.r.t. a TBox $\mathcal{T}$ ), denoted $c l_{\mathcal{T}}(\mathcal{A})$, is the set of all ABox assertions $\alpha$ such that $\mathcal{T} \cup \mathcal{A}=\alpha$. It is easy to see that in DL-Lite, $c l(\mathcal{T})$ and $c l_{\mathcal{T}}(\mathcal{A})$ can be computed in quadratic time in the size of $\mathcal{T}$ (and $\mathcal{A}$ ). In our work we assume that TBoxes and ABoxes are closed, i.e., equal to their deductive closure.

The DL-Lite family has nice computational properties, for example, KB satisfiability has polynomial-time complexity in the size of the TBox and logarithmic-space complexity in the size of the ABox [19, 23].

\section{Knowledge Expansion and Contraction Framework}

In this section, we first present our logical formalism of knowledge evolution, then introduce our evolution postulates, and finally relate our postulates to the well-known AGM postulates.

\subsection{Logical Formalism}

Consider a setting in which we have a knowledge base $\mathcal{K}=$ $(\mathcal{T}, \mathcal{A})$ developed by knowledge engineers. The KB $\mathcal{K}$ needs to be modified and a knowledge base $\mathcal{N}$ contains information about the modification. Intuitively, we are interested in two scenarios that can be described as follows:

- $\mathcal{K}$ is missing information captured in $\mathcal{N}$, and this new information $\mathcal{N}$ should be incorporated in $\mathcal{K}$, that is, $\mathcal{K}$ should be expanded with $\mathcal{N}$.

- $\mathcal{K}$ contains a modeling error, $\mathcal{N}$ describes this error, and $\mathcal{K}$ is to be contracted by 'extracting' $\mathcal{N}$ from $\mathcal{K}$.

More practically, we want to develop evolution operators for both expansion and contraction of knowledge bases that take $\mathcal{K}$ and $\mathcal{N}$ as input and return, preferably in polynomial time, a $D L$-Lite $\mathrm{KB} \mathcal{K}^{\prime}$ that captures the evolution, and which we call the evolution of $\mathcal{K}$ under $\mathcal{N}$. As described above, we consider two evolution scenarios:

- ontology expansion, when $\mathcal{N}=\mathcal{N}_{e}$ represents the information that should hold in $\mathcal{K}^{\prime}=\mathcal{K}_{e}^{\prime}$, and

- ontology contraction, when $\mathcal{N}=\mathcal{N}_{c}$ defines the information that should not hold in $\mathcal{K}^{\prime}=\mathcal{K}_{c}^{\prime}$.

Our general assumption about the framework is the following. We assume that both pieces of the new information, $\mathcal{N}_{e}$ and $\mathcal{N}_{c}$, are "prepared" to evolution, which means that $\mathcal{N}_{e}$ is

\footnotetext{
${ }^{4}$ Coherence is often called full satisfiability.
} 
coherent and $\mathcal{N}_{c}$ does not include tautologies. Indeed, if $\mathcal{N}_{e}$ is not coherent, this means that the information in $\mathcal{N}_{e}$ is not true and thus, before incorporating it into $\mathcal{K}$, it is necessary to resolve issues with $\mathcal{N}_{e}$ itself. If $\mathcal{N}_{c}$ contains tautological axioms, then it is clearly impossible to retract this knowledge from $\mathcal{K}$.

Additionally, apart from

1. KB evolution, as described above,

we distinguish two additional, special types of evolution:

2. TBox evolution, where $\mathcal{N}$ consists of TBox assertions only, and

3. ABox evolution, which satisfies the following conditions:

- the TBox of $\mathcal{K}^{\prime}$ should be equivalent to $\mathcal{T}$,

- $\mathcal{N}$ consists of ABox assertions only, and

- in the case of expansion, $\mathcal{T} \cup \mathcal{N}$ is coherent.

Intuitively, ABox evolution corresponds to the case where the TBox $\mathcal{T}$ of $\mathcal{K}$ is developed by domain specialists, does not contain wrong information, and should be preserved, while $\mathcal{N}$ is a collection of facts.

We now illustrate these definitions on the following example.

Example 3.1 (Running Example). Consider a KB where the structural knowledge is that wives (concept Wife) are exactly those individuals who have husbands (role HasHusband) and that some wives are employed (concept EmpWife). Bachelors (concept Bachelor) cannot be husbands. Priests (concept Priest) are clerics (concept $\mathrm{Cleric}$ ) and clerics are bachelors. Both clerics and wives are receivers of rent subsidies (concept Renter). We also know that adam and bob are priests, mary is a wife who is employed and her husband is john. Also, carl is a catholic minister (concept Minister).

This knowledge can be expressed in $D L$ - $_{\text {Lite }} \mathcal{F}$ by the KB $\mathcal{K}_{e x}$, consisting of the following TBox $\mathcal{T}$ and $\mathrm{ABox} \mathcal{A}$ :

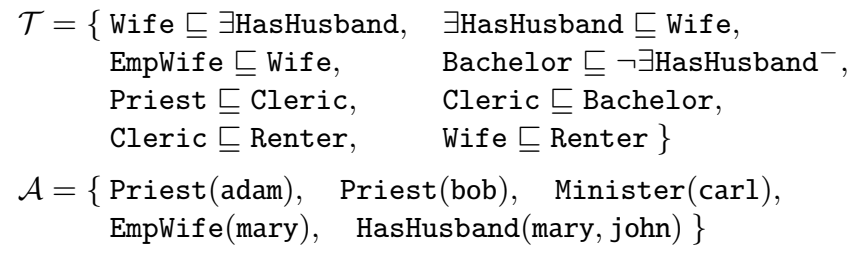

In the expansion scenario the new information $\mathcal{N}_{e}$ states that John is now a bachelor, that is, $\operatorname{Bachelor}(j o h n)$, and that catholic ministers are superiors of some religious orders and hence clerics, that is, Minister $\sqsubseteq$ Cleric. Therefore:

$$
\mathcal{N}_{e}=\{\text { Bachelor }(\text { john }), \quad \text { Minister } \sqsubseteq \text { Cleric }\} .
$$

In the contraction scenario, due to an economic crisis, rent subsidies got cancelled for priests, that is, $\mathcal{N}_{c}$ is

$$
\mathcal{N}_{c}=\{\text { Priest } \sqsubseteq \text { Renter }\} \text {. }
$$

Later on in the paper we will discuss how to incorporate such new knowledge $\mathcal{N}_{e}$ and $\mathcal{N}_{c}$ into the example $\mathrm{KB} \mathcal{K}_{e x}$.

\subsection{Postulates for Knowledge Base Evolution}

In the Semantic Web context, update/revision and erasure/contraction [7, 10], the classical understandings of ontology expansion and contraction, respectively, are too restrictive from the intuitive and formal perspective. Indeed, on the one hand the 'granularity' of knowledge changes when moving from propositional to Description Logics: the atomic statements of a DL, namely the ABox and TBox axioms, are more complex than the atoms of propositional logic. On the other hand, a set of propositional formulas makes sense, intuitively, if it is satisfiable, while a KB can be satisfiable, but incoherent, that is, one or more concepts are necessarily empty. Therefore, in the two following sections, we propose new postulates for expansion and contraction, to be adopted in the context of evolution on the Semantic Web.

Framework Postulates. The first two postulates describe the basic requirements of our framework. The first one is that evolution (both expansion and contraction) should preserve coherence:

E1: Expansion should preserve the coherence of the $\mathrm{KB}$, that is, if $\mathcal{K}$ is coherent, then so is $\mathcal{K}_{e}^{\prime}$.

C1: Contraction should not add any extraneous knowledge, that is, $\mathcal{K} \models \mathcal{K}_{c}^{\prime}$.

Observe that $\mathbf{C 1}$ does not say explicitly that contraction should preserve coherence; the latter, however, is implied. The next postulate formalises the idea that expansion should incorporate new knowledge:

E2: Expansion should entail all new knowledge, that is, $\mathcal{K}_{e}^{\prime}=\mathcal{N}_{e}$.

Unfortunately, there is no obvious way to say what a corresponding contraction postulate should be. Indeed, the most straightforward idea would be to say that $\mathcal{K}_{c}^{\prime} \not \models \mathcal{N}_{c}$, that is, there should exist a model of $\mathcal{K}_{c}^{\prime}$ that is not a model of $\mathcal{N}_{c}$. This requirement, however, leads to undesirable consequences as shown in the following example.

Example 3.2. Consider the $\mathrm{KB}$ consisting of the two axioms $A \sqsubseteq B$ and $C \sqsubseteq D$. Assume that we have learnt that both axioms are false and therefore the new information $\mathcal{N}_{c}$ consists of these two axioms. Observe that it is the case for both $\mathcal{K}_{1}^{\prime}=\{A \sqsubseteq B\}$ and $\mathcal{K}_{2}^{\prime}=\{C \sqsubseteq D\}$ that $\mathcal{K}_{i}^{\prime} \not \models \mathcal{N}_{c}$. However, intuitively, neither of them should be a result of contraction since either KB entails a piece of false information.

The example suggests that we need to make sure that $\mathcal{K}_{c}^{\prime}$ does not entail each axiom of $\mathcal{N}_{c}$. There are two alternatives:

C2: Contraction should not entail any piece of the new knowledge, i.e., $\mathcal{K}_{c}^{\prime} \not \models \alpha$ for all $\alpha \in \mathcal{N}_{c}$.

C2' $^{\prime}$ : Contraction should not entail the disjunction of the new knowledge, that is, $\mathcal{K}_{c}^{\prime} \forall \alpha_{1} \vee \cdots \vee \alpha_{n}$, where $\mathcal{N}_{c}=\left\{\alpha_{1}, \ldots, \alpha_{n}\right\}$. 
Note that in general $\mathbf{C 2}$ is strictly weaker than $\mathbf{C 2}{ }^{\prime}$ when $\mathcal{N}_{c}$ contains more than one axiom. That is, $\mathbf{C 2}$ ' entails $\mathbf{C 2}$, while the converse is not always the case 5 In our work we will focus rather on C2. Note also that most of our negative results hold already for contraction where $\mathcal{N}_{c}$ is a singleton and thus, when these two postulates coincide.

Basic Properties Postulates. The next postulates define the basic property that evolution operators should satisfy; namely, it states when no changes should be applied to the KB:

E3: Expansion with old information should not affect the $\mathrm{KB}$, that is, if $\mathcal{K} \models \mathcal{N}_{e}$, then $\mathcal{K}_{e}^{\prime} \equiv \mathcal{K}$.

C3: Contraction with conflicting information should not affect the $\mathrm{KB}$, that is, if $\mathcal{K} \not=\alpha$ for each $\alpha \in \mathcal{N}_{c}$, then $\mathcal{K}_{c}^{\prime} \equiv \mathcal{K}$.

Observe that we can also define the postulate $\mathbf{C} 3^{\prime}$, which is an alternative to $\mathbf{C 3}$, but based on $\mathbf{C 2}^{\prime}$.

The next two postulates define the preciseness of evolution:

E4: The union of $\mathcal{N}_{2 e}$ with the expansion of $\mathcal{K}$ with $\mathcal{N}_{1 e}$ implies the expansion of $\mathcal{K}$ with $\mathcal{N}_{1 e} \cup \mathcal{N}_{2 e}$.

C4: The union of $\mathcal{N}_{c}$ with the contraction of $\mathcal{K}$ with $\mathcal{N}_{c}$ implies $\mathcal{K}$.

Principle Postulates. The final two postulates represent evolution principles that are widely accepted in the literature. The first one is the principle of irrelevance of syntax:

E5: Expansion should not depend on the syntactical representation of knowledge, that is, if $\mathcal{K}_{1} \equiv \mathcal{K}_{2}$ and $\mathcal{N}_{1 e} \equiv \mathcal{N}_{2 e}$, then $\mathcal{K}_{1 e}^{\prime} \equiv \mathcal{K}_{2 e}^{\prime}$.

C5: Contraction should not depend on the syntactical representation of knowledge, that is, if $\mathcal{K}_{1} \equiv \mathcal{K}_{2}$ and $\mathcal{N}_{1 c} \equiv \mathcal{N}_{2 c}$, then $\mathcal{K}_{1 c}^{\prime} \equiv \mathcal{K}_{2 c}^{\prime}$.

Also, the so-called principle of minimal change is widely accepted in the literature [7, 9, 10]:

The change to $\mathcal{K}$ should be minimal, that is, $\mathcal{K}_{e}^{\prime}$ and $\mathcal{K}_{c}^{\prime}$ are minimally different from $\mathcal{K}$.

However, there is no general agreement on how to define this minimality and the current belief is that there is no general notion of minimality that will "do the right thing" under all circumstances [9]. In this work we will follow this belief and we will incorporate some suitable notion of minimality into each evolution semantics we introduce.

\subsection{Connection to AGM Postulates}

In this section we discuss the connection between our postulates and the AGM postulates of Alchourrón et al. [24]. The

\footnotetext{
${ }^{5}$ The converse holds, however, for DL-Lite. This is a direct consequence of Theorem 4.2
}

AGM approach has strongly influenced the formulation of postulates by Katsuno and Mendelzon in [7]. Given a (propositional) knowledge base $\psi$ and a sentence $\mu$, then $\psi \circ \mu$ denotes the revision of $\psi$ by $\mu$; that is, the new knowledge base obtained by adding new knowledge $\mu$ to the old knowledge base $\psi$. The following are the AGM postulates for revision:

(P+1) $\psi \circ \mu$ implies $\mu$.

(P+2) If $\psi \wedge \mu$ is satisfiable, then $\psi \circ \mu \equiv \psi \wedge \mu$.

(P+3) If $\mu$ is satisfiable, then $\psi \circ \mu$ is also satisfiable.

(P+4) If $\psi_{1} \equiv \psi_{2}$ and $\mu_{1} \equiv \mu_{2}$, then $\psi_{1} \circ \mu_{1} \equiv \psi_{2} \circ \mu_{2}$.

(P+5) $(\psi \circ \mu) \wedge \varphi$ implies $\psi \circ(\mu \wedge \varphi)$.

(P+6) If $(\psi \circ \mu) \wedge \varphi$ is satisfiable, then $\psi \circ(\mu \wedge \varphi)$ implies $(\psi \circ \mu) \wedge \varphi$.

Observe that $(\mathbf{P}+\mathbf{1})$ corresponds to our postulate $\mathbf{E 2},(\mathbf{P}+\mathbf{3})$ to E1, $(\mathbf{P}+4)$ to $\mathbf{E 5}$, and $(\mathbf{P + 5})$ to E4. Note that we do not have a postulate corresponding to $(\mathbf{P + 6})$, and instead of one corresponding to $(\mathbf{P}+\mathbf{2})$, we have the strictly weaker postulate $\mathbf{E 3}{ }^{6}$ The reason is that $(\mathbf{P}+\mathbf{2})$ and $(\mathbf{P}+\mathbf{6})$ reflect the view of Alchourrón et al. on the Principle of Minimal Change; we, however, would like to study a broader class of operators than the one considered by Alchourrón et al., so we did not adapt $(\mathbf{P + 6})$ and weakened $(\mathbf{P + 2})$.

Now we turn to the AGM postulates for contraction. Given a (propositional) knowledge base $\psi$ and a sentence $\mu$, then $\psi \bullet \mu$ denotes the contraction of $\psi$ by $\mu$. The following are the AGM postulates for contraction:

(P-1) $\psi$ implies $\psi \bullet \mu$.

(P-2) If $\psi$ does not imply $\mu$, then $\psi \bullet \mu$ is equivalent to $\psi$.

(P-3) If $\mu$ is not a tautology, then $\psi \bullet \mu$ does not imply $\mu$.

(P-4) If $\psi_{1} \equiv \psi_{2}$ and $\mu_{1} \equiv \mu_{2}$, then $\psi_{1} \bullet \mu_{1} \equiv \psi_{2} \bullet \mu_{2}$.

(P-5) $(\psi \bullet \mu) \wedge \mu$ implies $\psi$.

Observe that (P-1) corresponds to $\mathbf{C 1},(\mathbf{P}-\mathbf{3})$ to $\mathbf{C 2},(\mathbf{P}-\mathbf{4})$ to $\mathbf{C 5}$, and (P-5) to $\mathbf{C 4}$. Similarly to the case of expansion, we have substituted (P-2) with the weaker postulate $\mathbf{C} 3:^{7}$

\section{Model-based Approaches to Evolution}

Among the candidate semantics for evolution operators proposed in the literature we study first the model-based approaches (MBAs) [9, 12, 19, 25]. The section is organized as follows. First, we define MBAs along several dimensions. Then, we show negative results for MBAs in the context of $D L$ Lite. Finally, we discuss conceptual problems of MBAs.

\footnotetext{
${ }^{6}$ Compare E3 with (U2) in [7]

${ }^{7}$ Compare $\mathbf{C 3}$ with (E2) in [7].
} 


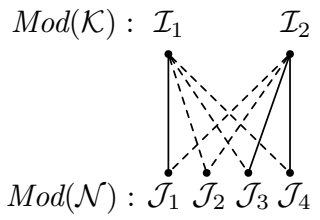

Figure 1: Model-based evolution semantics: example

\subsection{Definition of Model-based Approaches to Evolution}

We first define model-based expansion and then proceed to contraction.

Model-based Expansion. In MBAs, the result of the expansion of a KB $\mathcal{K}$ w.r.t. new knowledge $\mathcal{N}$ is a set $\mathcal{K} \circ \mathcal{N}$ of models. The general idea of MBAs is to choose as the result of evolution some models of $\mathcal{N}$ depending on their distance to the models of $\mathcal{K}$. Katsuno and Mendelzon [7] considered two ways of choosing these models of $\mathcal{N}$.

In the first one, which we call local, the idea is to go over all models $\mathcal{I}$ of $\mathcal{K}$ and for each $\mathcal{I}$ to take those models $\mathcal{J}$ of $\mathcal{N}$ that are minimally distant from $\mathcal{I}$. Formally,

$$
\mathcal{K} \circ_{\mathbb{L}} \mathcal{N}=\bigcup_{\mathcal{I} \in \operatorname{Mod}(\mathcal{K})} \underset{\mathcal{J} \in \mathcal{M}}{\arg \min } \operatorname{dist}(\mathcal{I}, \mathcal{J})
$$

where $(i) \operatorname{dist}(\cdot, \cdot)$ is a function that varies from approach to approach, and whose range is a partially ordered domain, (ii) arg min stands for the argument of the minimum, that is, in our case, the set of interpretations $\mathcal{J}$ for which the value of $\operatorname{dist}(\mathcal{I}, \mathcal{J})$ reaches a minimum given $\mathcal{I}$, and (iii) $\mathcal{M}$ is equal to $\operatorname{Mod}(\mathcal{N})$ in the case of $\mathrm{KB}$ evolution, or $\operatorname{Mod}(\mathcal{T} \cup \mathcal{N})$ in the case of ABox evolution. The distance function dist commonly takes as values either numbers or subsets of some fixed set, and the minimum is defined according to the partial order over its range.

In the second way, called global, the idea is to choose those models of $\mathcal{N}$ that are minimally distant from the entire set of models of $\mathcal{K}$. Formally,

$$
\mathcal{K} \circ_{\mathbb{G}} \mathcal{N}=\underset{\mathcal{J} \in \mathcal{M}}{\arg \min } \operatorname{dist}(\operatorname{Mod}(\mathcal{K}), \mathcal{J})
$$

where $\operatorname{dist}(\operatorname{Mod}(\mathcal{K}), \mathcal{J})=\min _{\mathcal{I} \in \operatorname{Mod}(\mathcal{K})} \operatorname{dist}(\mathcal{I}, \mathcal{J})$ and $\mathcal{M}$ is as in the previous case. Note that the minimum need not be unique, e.g., if distances are measured in terms of sets. Then the distance between $\operatorname{Mod}(\mathcal{K})$ and $\mathcal{J}$ is the set of all minimal distances $\operatorname{dist}(\mathcal{I}, \mathcal{J})$ between elements $\mathcal{I}$ of $\operatorname{Mod}(\mathcal{K})$ and $\mathcal{J}$.

To get a better intuition of local semantics, consider Figure 1 . which depicts two models $\mathcal{I}_{1}$ and $\mathcal{I}_{2}$ of $\mathcal{K}$, and four interpretations $\mathcal{J}_{1}, \ldots, \mathcal{J}_{4}$ that satisfy $\mathcal{N}$. The distance between $\mathcal{I}_{i}$ and $\mathcal{J}_{j}$ is represented by the shape of the line connecting them: solid lines correspond to minimal distances, and dashed ones to distances that are not minimal. In this case, $\mathcal{J}_{1}$ is in $\mathcal{K} \circ_{\mathbb{L}} \mathcal{N}$, because it is minimally distant from $\mathcal{I}_{1}$, and $\mathcal{J}_{3}$ and $\mathcal{J}_{4}$ are in $\mathcal{K} \circ_{\mathbb{L}} \mathcal{N}$, because they are minimally distant from $\mathcal{I}_{2}$.
Model-based Contraction. In the literature, contraction in the DL setting received much less attention than expansion. The general view on contraction, which originates from the ideas of contraction in propositional logic (see Section 3.3), is that the resulting set of models can be divided into two parts: first, the models of the original $\mathrm{KB} \mathcal{K}$ (cf. C4), and second, interpretations that falsify the axioms of $\mathcal{N}$ (cf. C2) and that are minimally distant from the models of $\mathcal{K}$. Following this view, we define local and global model-based contraction operators as follows:

$$
\begin{aligned}
& \mathcal{K} \bullet_{\mathbb{L}} \mathcal{N}=\operatorname{Mod}(\mathcal{K}) \cup \bigcup_{\varphi \in \mathcal{N}} \bigcup_{\mathcal{I} \in \operatorname{Mod}(\mathcal{K})} \underset{\mathcal{J} \in \mathcal{M}_{\neg \varphi}}{\arg \min } \operatorname{dist}(\mathcal{I}, \mathcal{J}), \\
& \mathcal{K} \bullet_{\mathbb{G}} \mathcal{N}=\operatorname{Mod}(\mathcal{K}) \cup \bigcup_{\varphi \in \mathcal{N}} \underset{\mathcal{J} \in \mathcal{M}_{\neg \varphi}}{\arg \min \operatorname{dist}(\operatorname{Mod}(\mathcal{K}), \mathcal{J}),}
\end{aligned}
$$

where $\mathcal{M}_{\neg \varphi}$ is equal to $(i)\{\mathcal{J} \mid \mathcal{J} \forall \varphi$ in the case of $\mathrm{KB}$ evolution, or (ii) $\{\mathcal{J} \mid \mathcal{J} \in \operatorname{Mod}(\mathcal{T})$ and $\mathcal{J} \not \forall \varphi\}$ in the case of ABox evolution. Observe that the second part of each definition, which builds the part of $\mathcal{K} \bullet \mathcal{N}$ that falsifies $\mathcal{N}$, can be defined differently (e.g., the condition in the definition of $\mathcal{M}_{\neg \varphi}$ could be $\mathcal{J} \forall \bigvee_{\varphi \in \mathcal{N}} \varphi$, which corresponds to $\mathbf{C 2}^{\prime}$ ) or in a more general way (e.g., see [26]). We argue, however, that most model-based contraction operators satisfying our postulates coincide with one of our operators in the case when $|\mathcal{N}|=1$, and since all of our negative results hold already for this case, they also apply to these other definitions.

Three-dimensional Space of MBAs. The classical MBAs have been developed for propositional theories. In this context, an interpretation can be identified with the set of propositional atoms that it makes true, and two distance functions have been introduced. They are respectively based on the symmetric difference and on the cardinality of the symmetric difference of interpretations, namely

$$
\operatorname{dist}_{\subseteq}(\mathcal{I}, \mathcal{J})=\mathcal{I} \ominus \mathcal{J} \quad \text { and } \quad \operatorname{dist}_{\#}(\mathcal{I}, \mathcal{J})=|\mathcal{I} \ominus \mathcal{J}|
$$

where the symmetric difference $\mathcal{I} \ominus \mathcal{J}$ of two sets $\mathcal{I}$ and $\mathcal{J}$ is defined as $\mathcal{I} \ominus \mathcal{J}=(\mathcal{I} \backslash \mathcal{J}) \cup(\mathcal{J} \backslash \mathcal{I})$. Distances under dist $\subset$ are sets and are compared by set inclusion, that is, $\operatorname{dist}_{\subseteq}\left(\mathcal{I}_{1}, \mathcal{J}_{1}\right) \leq$ $\operatorname{dist}_{\subseteq}\left(\mathcal{I}_{2}, \mathcal{J}_{2}\right)$ iff dist $_{\subseteq}\left(\mathcal{I}_{1}, \mathcal{J}_{1}\right) \subseteq$ dist $\subseteq_{\subseteq}\left(\mathcal{I}_{2}, \mathcal{J}_{2}\right)$. Distances under dist $_{\#}$ are natural numbers and are compared in the standard way.

One can extend these distances to DL interpretations in two different ways. One way is to consider interpretations $\mathcal{I}, \mathcal{J}$ as sets of atoms. Then $\mathcal{I} \ominus \mathcal{J}$ is again a set of atoms and we can define distances as in Equation (2). We denote these distances as $\operatorname{dist}_{\subseteq}^{a}(\mathcal{I}, \mathcal{J})$ and $\operatorname{dist}_{\#}^{a}(\mathcal{I}, \mathcal{J})$, respectively. While in the propositional case distances are always finite, note that this may not be the case for DL interpretations that are infinite. Another way is to define distances at the level of the concept and role symbols in the signature $\Sigma$ underlying the interpretations:

$$
\begin{aligned}
& \operatorname{dist}_{\subseteq}^{s}(\mathcal{I}, \mathcal{J})=\left\{S \in \Sigma \mid S^{\mathcal{I}} \neq S^{\mathcal{J}}\right\}, \quad \text { and } \\
& \operatorname{dist}_{\#}^{s}(\mathcal{I}, \mathcal{J})=\left|\left\{S \in \Sigma \mid S^{\mathcal{I}} \neq S^{\mathcal{J}}\right\}\right| .
\end{aligned}
$$

Summing up across the different possibilities, we have three dimensions, which give eight possibilities to define a semantics 


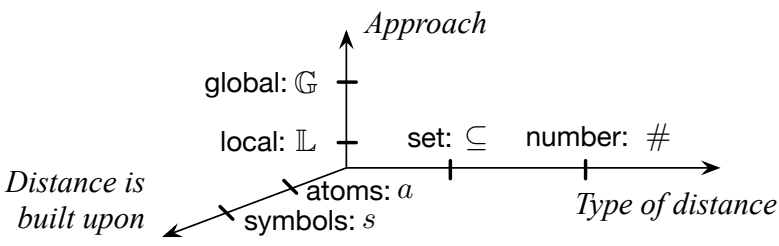

Figure 2: Three-dimensional space of model-based evolution semantics

of evolution according to MBAs by choosing (as depicted in Figure 2]:

1. the local or the global approach,

2. atoms or symbols for defining distances, and

3. set inclusion or cardinality to compare symmetric differences.

We denote each of these eight possibilities by a combination of three symbols, indicating the choice in each dimension. By $\mathbb{L}$ we denote local and by $\mathbb{G}$ global semantics. We attach the superscripts $a$ or $s$ to indicate whether distances are defined in terms of atoms or symbols, respectively. And we use the subscripts $\subseteq$ or \# to indicate whether distances are compared in terms of set inclusion or cardinality, respectively. For example, $\mathbb{L}_{\#}^{a}$ denotes the local semantics where the distances are expressed in terms of cardinality of sets of atoms.

Considering that in the propositional case a distinction between atom and symbol-based semantics is meaningless, we can also use our notation, without superscripts, to identify MBAs in that setting. Interestingly, the two classical local MBAs proposed by Winslett [9] and Forbus [27] correspond, respectively, to $\mathbb{L}_{\subset}$, and $\mathbb{L}_{\#}$, while the one by Borgida [28] is a variant of $\mathbb{L}_{\subseteq}$. The two classical global MBAs proposed by Satoh [29] and Dalal [30] correspond respectively to $\mathbb{G}_{\subseteq}$, and $\mathbb{G}_{\#}$.

Next, we show that these semantics satisfy the evolution postulates defined in Section 3.2.

Proposition 4.1. For $\mathbb{X} \in\{\mathbb{G}, \mathbb{L}\}, y \in\{s, a\}$ and $z \in\{\subseteq, \#\}$,

- the expansion operator $\circ_{\mathbb{X}}^{y}$ satisfies $\boldsymbol{E 1 - E 5}$;

- the contraction operator $\bullet_{\mathbb{X}}^{y}$ satisfies $\boldsymbol{C 1}-\mathbf{C 5}$.

Proof. The claim for E1, E2, E5, C1, C2, and C5 follows directly from the definitions of the operators. E3 follows from the observation that if $\mathcal{K} \models \mathcal{N}$, then $\mathcal{M}$ in the definition of the operators coincides with $\operatorname{Mod}(\mathcal{K})$, and thus each model of $\mathcal{M}$ is minimally distant from itself. $\mathbf{C 3}$ follows from the observation that if $\mathcal{K} \forall \alpha$ for each $\alpha \in \mathcal{N}$, then the models of $\mathcal{M}_{\neg \varphi}$ minimally distant from some model $\mathcal{I}$ of $\mathcal{K}$ (resp., from $\mathcal{K}$ ) are exactly those models of $\mathcal{K}$ that falsify some $\varphi$. Regarding E4, the claim is trivial if $\left(\mathcal{K} \circ_{\mathbb{L}_{z}^{y}} \mathcal{N}_{1}\right) \cup \mathcal{N}_{2}$ is not satisfiable. If it is satisfiable, then observe that if $\mathcal{I} \in$

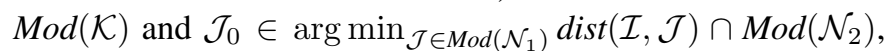
then $\mathcal{J}_{0} \in \arg \min _{\mathcal{J} \in \operatorname{Mod}\left(\mathcal{N}_{1} \cup \mathcal{N}_{2}\right)} \operatorname{dist}(\mathcal{I}, \mathcal{J})$. The proofs for the case of $\mathbb{G}_{z}^{y}$ and the case of ABox expansion are similar. Finally, C4 follows from the following observation: if $\mathcal{J}_{0} \in\left(\mathcal{K} \bullet_{\mathbb{L}_{z}^{y}} \mathcal{N}\right) \cup \mathcal{N}$, Then $\mathcal{J}_{0} \in\left(\operatorname{Mod}(\mathcal{K}) \cup \mathcal{M}^{\prime}\right) \cap \operatorname{Mod}(\mathcal{N})$, where $\mathcal{M}^{\prime}=\arg \min _{\mathcal{J} \in \mathcal{M}_{\neg \varphi}} \operatorname{dist}(\mathcal{I}, \mathcal{J})$ for some model $\mathcal{I}$ of $\mathcal{K}$ and some $\varphi \in \mathcal{N}$. From $\mathcal{J}_{0} \in \operatorname{Mod}(\mathcal{N})$ we conclude that $\mathcal{J}_{0} \models \varphi$ and consequently $\mathcal{J}_{0} \in \operatorname{Mod}(\mathcal{K}) \backslash \mathcal{M}^{\prime}$, which proves the claim. The proof for the case of $\mathbb{G}_{z}^{y}$ is similar.

Under each of our eight semantics, expansion results in a set of interpretations. In the propositional case, each set of interpretations over finitely many symbols can be captured by a formula whose models are exactly those interpretations. In the case of DLs, this is not necessarily the case, since on the one hand, a KB might have infinitely many infinite models and, on the other hand, logics may lack some connectives like disjunction or negation. Thus, a natural problem arising in the case of DLs is the expressibility problem.

Let $\mathcal{D}$ be a DL and $\mathbb{M}$ one of the eight MBAs introduced above. We say that $\mathcal{D}$ is closed under expansion for $\mathbb{M}$ (or that expansion w.r.t. $\mathbb{M}$ is expressible in $\mathcal{D}$ ), if for all $\mathrm{KBs} \mathcal{K}$ and $\mathcal{N}$ written in $\mathcal{D}$, there is a $\mathrm{KB} \mathcal{K}^{\prime}$ also written in $\mathcal{D}$ such that $\operatorname{Mod}\left(\mathcal{K}^{\prime}\right)=\mathcal{K} \circ_{\mathbb{M}} \mathcal{N}$. Analogously, we say that $\mathcal{D}$ is closed under contraction for $\mathbb{M}$ (or that contraction w.r.t. $\mathbb{M}$ is expressible in $\mathcal{D}$ ), if for all $\mathrm{KBs} \mathcal{K}$ and $\mathcal{N}$ written in $\mathcal{D}$, there is a $\mathrm{KB} \mathcal{K}^{\prime}$ also written in $\mathcal{D}$ such that $\operatorname{Mod}\left(\mathcal{K}^{\prime}\right)=\mathcal{K} \bullet \mathbb{M} \mathcal{N}$. We study now whether $D L$-Lite $\mathcal{F}_{\mathcal{R}}$ is closed under evolution w.r.t. the various semantics.

\subsection{Inexpressibility of Model-based Approaches}

We show now that both expansion and contraction, w.r.t. the introduced semantics are inexpressible in $D L$-Lite $\mathcal{F} \mathcal{R}$. Moreover, all our inexpressibility results hold already for TBox evolution, and for five of the eight considered semantics we show it for ABox evolution.

The key observation underlying these results is that, on the one hand, the principle of minimal change often introduces implicit disjunction in the resulting KB. On the other hand, DL-Lite ${ }_{\mathcal{F} \mathcal{R}}$ can be embedded into a slight extension of Horn logic [31] and therefore does not allow one to express genuine disjunction. Technically, this can be expressed by saying that every $D L$-Lite $_{\mathcal{F} \mathcal{R}} \mathrm{KB}$ that entails a disjunction of $D L$-Lite $_{\mathcal{F} \mathcal{R}}$ assertions entails one of the disjuncts. The theorem below gives a contrapositive formulation of this statement. Although $D L$ Lite $_{\mathcal{F} \mathcal{R}}$ does not have a disjunction operator, by abuse of notation we write $\mathcal{J} \models \varphi \vee \psi$ as a shorthand for " $\mathcal{J} \models \varphi$ or $\mathcal{J} \models \psi$ ", for DL-Lite $\mathcal{F} \mathcal{R}$ assertions $\varphi$ and $\psi$.

Theorem 4.2. Let $\mathcal{M}$ be a set of interpretations. Suppose there are DL-Lite assertions $\varphi$, $\psi$ such that

1. $\mathcal{J} \models \varphi \vee \psi$ for every $\mathcal{J} \in \mathcal{M}$, and

2. there are $\mathcal{J}_{\varphi}, \mathcal{J}_{\psi} \in \mathcal{M}$ such that $\mathcal{J}_{\varphi} \not \models \varphi$ and $\mathcal{J}_{\psi} \not \forall \psi$. Then, there is no DL-Lite $\mathcal{F}_{\mathcal{R}} K B \mathcal{K}$ such that $\mathcal{M}=\operatorname{Mod}(\mathcal{K})$.

Proof. We prove the theorem by contradiction. Assume there exists a $D L$-Lite ${ }_{\mathcal{F} R} \mathrm{~KB} \mathcal{K}$ such that for every model $\mathcal{J}$ of $\mathcal{K}$ we have $\mathcal{J}=\varphi \vee \psi$, but $\mathcal{K} \not=\varphi$ and $\mathcal{K} \not \psi \psi$.

We distinguish the two cases (1) $\varphi$ and $\psi$ are membership assertions, and (2) $\varphi$ is an arbitrary assertion while $\psi$ is an inclusion or functionality assertions.

Case 1. This part of the proof relies on a result by Calvanese et al. [15] who showed that for every satisfiable $D L$ Lite $_{\mathcal{F} \mathcal{R}} \mathrm{KB} \mathcal{K}$ there exists a model $\mathcal{I}_{\mathcal{K}}$, the canonical model 
of $\mathcal{K}$, that can be homomorphically mapped to every other model of $\mathcal{K}$. Formally, for every model $\mathcal{J}$ there is a mapping $h: \Delta \rightarrow \Delta$ such that $(i) h(a)=a$ for every constant $a$ appearing in $\mathcal{K}$, (ii) $h\left(A^{\mathcal{I}_{\mathcal{K}}}\right) \subseteq A^{\mathcal{J}}$ for every atomic concept $A$, and (iii) $h\left(P^{\mathcal{I}_{\mathcal{K}}}\right) \subseteq P^{\mathcal{J}}$ for every atomic role $P$. In essence, the canonical model is constructed by chasing the ABox of $\mathcal{K}$ with the positive inclusion assertions in the TBox of $\mathcal{K}$, that is, the inclusion assertions without negation sign. Intuitively, the homomorphism $h$ exists because every model $\mathcal{J}$ of $\mathcal{K}$ satisfies these assertions, and therefore all atoms introduced by the chase into $\mathcal{I}_{\mathcal{K}}$ have a corresponding atom in $\mathcal{J}$. (Technically, there is a slight difference between our definition of interpretations and the one in [15], as we assume that all interpretations share the same domain, while domains can be arbitrary non-empty sets in [15]. The argument in [15], however, can be carried over in a straightforward way to our setting.)

Now, for the canonical model $\mathcal{I}_{\mathcal{K}}$ of $\mathcal{K}$ we have $\mathcal{I}_{\mathcal{K}} \models \varphi \vee \psi$. Then one of $\varphi$ and $\psi$ is satisfied by $\mathcal{I}_{\mathcal{K}}$, say $\varphi$. However, since $\mathcal{I}_{\mathcal{K}}$ is canonical, $\varphi$ is also satisfied by every other model $\mathcal{J}$ of $\mathcal{K}$, due to the existence of a homomorphism from $\mathcal{I}_{\mathcal{K}}$ to $\mathcal{J}$. For example, if $\varphi=A(a)$, then $\mathcal{I}_{\mathcal{K}} \models A(a)$ implies $a \in A^{\mathcal{I}_{\mathcal{K}}}$, which implies $a=h(a) \in h\left(A^{\mathcal{I}_{\mathcal{K}}}\right) \subseteq A^{\mathcal{J}}$, that is, $\mathcal{J} \models A(a)$. For other kinds assertions, a similar argument applies. This contradicts the assumption that there exists a $\mathcal{J}_{\varphi}$ that falsifies $\varphi$.

Case 2. Let $\mathcal{K}=\mathcal{T} \cup \mathcal{A}$. The argument for this case will be based on the fact that the disjoint union of a model of $\mathcal{K}$ and a model of the $\mathcal{T}$ is again a model of $\mathcal{K}$, while the disjoint union of a counterexample for $\varphi$ and a counterexample for $\psi$ is a counterexample for both. In order to formalise this idea we need some notation and simple facts as a preparation.

Given two interpretations $\mathcal{I}_{1}, \mathcal{I}_{2}$, their union $\mathcal{I}_{1} \cup \mathcal{I}_{2}$ is the interpretation defined by $A^{\mathcal{I}_{1} \cup \mathcal{I}_{2}}=A^{\mathcal{I}_{1}} \cup A^{\mathcal{I}_{2}}$ for every primitive concept $A$ and $P^{\mathcal{I}_{1} \cup \mathcal{I}_{2}}=P^{\mathcal{I}_{1}} \cup P^{\mathcal{I}_{2}}$ for every primitive role $P$. From the definition it follows also for all concepts of the form $B=\exists R$, where $R$ is one of $P$ or $P^{-}$, that $B^{\mathcal{I}_{1} \cup \mathcal{I}_{2}}=B^{\mathcal{I}_{1}} \cup B^{\mathcal{I}_{2}}$.

We define the support set of $\mathcal{I}$ as the set of constants that occur in the interpretation of some atomic concept or role under $\mathcal{I}$. If $\mathcal{I}_{1}, \mathcal{I}_{2}$ have disjoint support sets, we denote their union also as $\mathcal{I}_{1} \uplus \mathcal{I}_{2}$ and speak of a disjoint union.

Let $\alpha$ be an inclusion or functionality assertion, let $\beta$ be a membership assertion, and let $\mathcal{I}_{1}, \mathcal{I}_{2}$ be interpretations with disjoint support. Then the following statements are straightforward to check:

(i) $\mathcal{I}_{1} \uplus \mathcal{I}_{2} \models \alpha$ iff $\mathcal{I}_{1}=\alpha$ and $\mathcal{I}_{2}=\alpha$;

(ii) if the support set of $\mathcal{I}_{2}$ is disjoint from the set of constants of $\beta$, then $\mathcal{I}_{1} \uplus \mathcal{I}_{2} \models \beta$ iff $\mathcal{I}_{1} \models \beta$;

(iii) $\mathcal{I}_{1} \uplus \mathcal{I}_{2}$ is a model of $\mathcal{T}$ iff $\mathcal{I}_{1}$ and $\mathcal{I}_{2}$ are both models of $\mathcal{T}$;

(iv) if the support set of $\mathcal{I}_{2}$ is disjoint from the set of constants of $\mathcal{K}$, then $\mathcal{I}_{1} \uplus \mathcal{I}_{2}$ is a model of $\mathcal{K}$ iff $\mathcal{I}_{1}$ is a model of $\mathcal{K}$ and $\mathcal{I}_{2}$ is a model of $\mathcal{T}$.

Here, (iii) follows from (i), and (iv) from (ii) and (iii). The assumption about the disjoint support sets is needed in (i), (iii) and (iv) to guarantee that negative inclusion assertions and functionality assertions continue to hold in $\mathcal{I}_{1} \uplus \mathcal{I}_{2}$. In addition, the assumptions about the disjointness of constant sets and the support set of $\mathcal{I}_{2}$ in (ii) and (iv) are needed to guarantee that $\mathcal{I}_{2}$ has no influence on the satisfaction of membership assertions.

Next, we introduce a technique to create disjoint variants of interpretations by moving their support sets with injective functions. If $f: \Delta \rightarrow \Delta$ is an injective mapping, then the image of $\mathcal{I}$ under $f$ is the interpretation $\mathcal{I}^{f}$ satisfying $A^{\mathcal{I}^{f}}=f\left(A^{\mathcal{I}}\right)$ for every atomic concept $A$ and $P^{\mathcal{I}^{f}}=f\left(P^{\mathcal{I}}\right)$ for every atomic role $P$. If $K$ is a set of constants, we say that $f$ respects $K$ if $f(a)=a$ for every constant $a \in K$.

Let $\alpha$ be an inclusion or functionality assertion, $\beta$ a membership assertion, and $\mathcal{I}$ an interpretation. Then the following statements are straightforward to check:

(v) $\mathcal{I} \models \alpha$ if and only if $\mathcal{I}^{f} \models \alpha$;

(vi) if $f$ respects the constants occurring in $\beta$, then $\mathcal{I} \models \beta$ iff $\mathcal{I}^{f}=\beta$;

(vii) $\mathcal{I}$ is a model of $\mathcal{T}$ iff $\mathcal{I}^{f}$ is a model of $\mathcal{T}$;

(viii) if $f$ respects the constants of $\mathcal{K}$, then $\mathcal{I}$ is a model of $\mathcal{K}$ iff $\mathcal{I}^{f}$ is a model of $\mathcal{K}$.

Note that the injectivity assumption is needed for $\mathcal{I}^{f}$ to satisfy negative inclusion assertions and functionality assertions if $\mathcal{I}$ does.

Now, suppose that $\varphi$ is an arbitrary assertion and that $\psi$ is an inclusion or functionality assertion. Moreover, let $\mathcal{J}_{\varphi}, \mathcal{J}_{\psi}$ be models of $\mathcal{K}$ such that $\mathcal{J}_{\varphi} \not \models \varphi$ and $\mathcal{J}_{\psi} \not \models \psi$. To create disjoint variants of these interpretations, we choose injective mappings $f, g: \Delta \rightarrow \Delta$ such that $f$ respects the constants of $\mathcal{K}$ and $\varphi$, and $f(\Delta) \cap g(\Delta)=\emptyset$. Clearly, such mappings always exist. From the facts about images of interpretations, we conclude that

1. $\mathcal{J}_{\varphi}^{f}$ is a model of $\mathcal{K}$ and $\mathcal{J}_{\varphi}^{f} \not=\varphi$;

2. $\mathcal{J}_{\psi}^{g}$ is a model of $\mathcal{T}$ and $\mathcal{J}_{\psi}^{g} \not \models \psi$;

3. $\mathcal{J}_{\varphi}^{f}$ and $\mathcal{J}_{\psi}^{g}$ have disjoint support sets.

Hence, for $\mathcal{J}=\mathcal{J}_{\varphi}^{f} \uplus \mathcal{J}_{\psi}^{g}$ we have that $\mathcal{J}$ is a model of $\mathcal{K}$ and $\mathcal{J}$ falsifies both $\varphi$ and $\psi$. This contradicts the assumption that every model of $\mathcal{K}$ satisfies one of $\varphi$ or $\psi$.

\subsubsection{KB Evolution}

In this part we show that $D L-$ Lite $_{\mathcal{F R}}$ is not closed under TBox evolution (both expansion and contraction) for any of the introduced MBAs. We start with the following example that illustrates the issue.

Example 4.3. Consider the $\mathrm{KB} \mathcal{K}_{e x}$ of our running example and assume that the new information $\mathcal{N}_{T}=\{$ Wife $\sqsubseteq$ $\neg$ Renter $\}$ arrived. We explore expansion w.r.t. the semantics $\mathbb{G}_{\#}^{s}$, which counts for how many symbols the interpretation changes.

Consider three assertions, (derived) from $\mathcal{K}$, that are essential for this example: EmpWife $\sqsubseteq$ Wife, EmpWife $\sqsubseteq$ Renter, 
and EmpWife(mary). One easily verifies that the minimum of $\operatorname{dist}_{\#}^{s}(\mathcal{I}, \mathcal{J})$ for $\mathcal{I} \in \operatorname{Mod}(\mathcal{K})$ and $\mathcal{J} \in \operatorname{Mod}\left(\mathcal{N}_{T}\right)$ is 1 , since, intuitively, we can turn a model of $\mathcal{K}$ into a model of $\mathcal{N}_{T}$ by dropping mary either from Wife or from Renter. Let $\mathcal{J} \in \mathcal{K} \circ_{\mathbb{G}^{s}} \mathcal{N}_{T}$. Then there exists $\mathcal{I} \in \operatorname{Mod}(\mathcal{K})$ such that $\operatorname{dist}_{\#}^{s}(\mathcal{I}, \mathcal{J})^{\#}=1$. Hence, there is only one symbol $S \in$ \{EmpWife, Wife, Renter\} whose interpretation has changed from $\mathcal{I}$ to $\mathcal{J}$, that is $S^{\mathcal{I}} \neq S^{\mathcal{J}}$. Observe that $S$ cannot be EmpWife. Otherwise, Wife and Renter would be interpreted identically under $\mathcal{I}$ and $\mathcal{J}$, and Wife and Renter would not be disjoint under $\mathcal{J}$, since mary is an instance of both, thus contradicting $\mathcal{N}_{T}$. Now, assume that Wife has not changed. Then $\mathcal{J}=$ EmpWife $\sqsubseteq$ Wife, since this held already for $\mathcal{I}$. However, $\mathcal{J} \not \models$ EmpWife $\sqsubseteq$ Renter, since mary $\in$ EmpWife $^{\mathcal{J}}$, but mary $\notin$ Renter $^{\mathcal{J}}$, due to the disjointness of Wife and Renter with respect to $\mathcal{J}$. Similarly, if we assume that Renter has not changed, it follows that $\mathcal{J} \models$ EmpWife $\sqsubseteq$ Renter, but $\mathcal{J} \not=$ EmpWife $\sqsubseteq$ Wife. By Theorem 4.2 we conclude that $\mathcal{K} \circ_{\mathbb{G}_{\#}^{s}} \mathcal{N}_{T}$ is not expressible in $D L$-Lite $\mathcal{F} \mathcal{R}$.

We now proceed to our first inexpressibility result, for $\mathrm{KB}$ expansion.

Theorem 4.4. DL-Lite FR $_{\mathcal{R}}$ is not closed under $K B$ expansion for $\mathbb{X}_{z}^{y}$, where $\mathbb{X} \in\{\mathbb{G}, \mathbb{L}\}, y \in\{s, a\}$, and $z \in\{\subseteq, \#\}$. Moreover, this holds already when both the initial $K B$ and the new information are written in DL-Lite core $_{\text {and }}$ the new information consists of a single TBox axiom.

Proof. The main idea of the proof is that evolution changes models in such a way that capturing them all would require to have a disjunction, which is impossible by Theorem 4.2. We generalize the idea of Example 4.3 where the inexpressibility of TBox expansion w.r.t. $\mathbb{G}_{\#}^{s}$ has already been shown.

To show inexpressibility of expansion w.r.t. all eight semantics, we consider the same fragment of our running example:

$$
\begin{aligned}
\mathcal{K}_{T} & =\{\text { EmpWife } \sqsubseteq \text { Wife }, \text { EmpWife } \sqsubseteq \text { Renter }\} \\
\mathcal{K}_{A} & =\{\text { EmpWife(mary })\} \\
\mathcal{N}_{T} & =\{\text { Wife } \sqsubseteq \neg \text { Renter }\}
\end{aligned}
$$

and $\mathcal{K}=\mathcal{K}_{T} \cup \mathcal{K}_{A}$.

We first consider expansion under global semantics. With an argument as in Example 4.3 , one verifies that there are models $\mathcal{I}$ of $\mathcal{K}$ where only mary is both a Wife and a Renter, and such models can be turned into models $\mathcal{J}$ of $\mathcal{N}_{T}$ by either dropping mary from the set of wives or from the set of renters. For these models we have

$$
\begin{aligned}
\text { - } \operatorname{dist}_{\subseteq}^{a}(\mathcal{I}, \mathcal{J}) & =\{\text { Wife }(\text { mary })\} \text { or } \\
\operatorname{dist}_{\subseteq}^{a}(\mathcal{I}, \mathcal{J}) & =\{\text { Renter }(\operatorname{mary})\} \\
\text { - } \operatorname{dist}_{\#}^{a}(\mathcal{I}, \mathcal{J}) & =1 ; \\
\text { - } \text { dist }_{\subseteq}^{s}(\mathcal{I}, \mathcal{J}) & =\{\text { Wife }\} \text { or } \text { dist }_{\subseteq}^{s}(\mathcal{I}, \mathcal{J})=\{\text { Renter }\} \\
\text { - } \text { dist }_{\#}^{s}(\mathcal{I}, \mathcal{J}) & =1 .
\end{aligned}
$$

Under each of the concerned semantics, these distances are minimal because smaller distances could only be 0 or the empty set, respectively, and interpretations with cardinality distance 0 or empty set-difference are identical. Hence, for every model $\mathcal{J} \in \mathcal{K} \circ_{\mathbb{G}_{z}^{y}} \mathcal{N}_{T}$ there is a model $\mathcal{I} \in \mathcal{K}$ that differs from $\mathcal{J}$ only in the interpretation of one concept, either Wife or Renter. It follows that (1) each such $\mathcal{J}$ either satisfies Wife(mary) or Renter(mary) and (2) there are $\mathcal{J}$ that satisfy one of the two assertions, but not the other. Thus, by Theorem 4.2, for none of the global semantics it is possible to express expansion in DL-Lite FR $_{\mathcal{R}}$.

Next, we turn to local semantics. The arguments used here are a slight variant of the ones above, taking into account the difference between the two kinds of semantics. We start with some $\mathcal{I} \in \operatorname{Mod}(\mathcal{K})$. In such a model, mary for sure is both a Wife and a Renter, but there may be further individuals that are instances of both of these concepts. Such an $\mathcal{I}$ can be turned into a model $\mathcal{J} \in \mathcal{N}_{T}$ by dropping for each individual $o \in \operatorname{Wife}^{\mathcal{I}} \cap \operatorname{Renter}^{\mathcal{I}}$ either the atom Wife $(o)$ or the atom Renter $(o)$.

With respect to the atom-based distances $d i s t_{\subseteq}^{a}$ and $d i s t_{\#}^{a}$, each $\mathcal{J}$ obtained in this way has minimal distance to $\mathcal{I}$. Moreover, these are the only models of $\mathcal{N}_{T}$ with minimal distance to $\mathcal{I}$ because further changes would increase the difference set and therefore the difference count.

With respect to the symbol-based distances $d i s t_{\subseteq}^{s}$ and $d i s t_{\#}^{s}$, a $\mathcal{J}$ obtained in this way is only minimal if the dropped atoms all have the same symbol. In this case we have again $\operatorname{dist}_{\subset}^{s}(\mathcal{I}, \mathcal{J})=\{$ Wife $\}$ or $\operatorname{dist}_{\subset}^{s}(\mathcal{I}, \mathcal{J})=\{$ Renter $\}$ and, correspondingly, dist $t_{\#}^{s}(\mathcal{I}, \mathcal{J})=1$. There are, however, further models of $\mathcal{J} \in \mathcal{N}_{T}$ with the same minimal distance to $\mathcal{I}$, namely those that in $\mathcal{J}$ interpret individuals as instances of Wife (or Renter, respectively) that in $\mathcal{I}$ were neither instances of Wife nor of Renter.

In summary, since $\mathcal{I}$ was chosen arbitrarily, we have seen again that (1) each $\mathcal{J} \in \mathcal{K} \circ_{\mathbb{L} z}^{y} \mathcal{N}_{T}$ either satisfies Wife(mary) or Renter (mary) and (2) there are $\mathcal{J}$ that satisfy one of the two assertions, but not the other. So, the conditions of Theorem 4.2 are satisfied and thus for none of the local semantics it is possible to express expansion in $D L-$ Lite $_{\mathcal{F} \mathcal{R}}$.

We now proceed to our second inexpressibility result, for KB contraction.

Theorem 4.5. DL-Lite ${ }_{\mathcal{F} \mathcal{R}}$ is not closed under $K B$ contraction for $\mathbb{X}_{z}^{y}$, where $\mathbb{X} \in\{\mathbb{G}, \mathbb{L}\}, y \in\{s, a\}$, and $z \in\{\subseteq, \#\}$. Moreover, this holds already when both the initial $K B$ and the new information are written in DL-Lite core $_{\text {and }}$ the new information consists of a single TBox axiom.

Proof. To show inexpressibility of contraction we consider another fragment of our running example:

$$
\begin{aligned}
\mathcal{K} & =\{\text { Priest } \sqsubseteq \text { Cleric, Cleric } \sqsubseteq \text { Renter }\} \\
\mathcal{N}_{T} & =\{\text { Priest } \sqsubseteq \text { Renter }\} .
\end{aligned}
$$

We first consider local semantics. To obtain $\mathcal{K} \bullet \mathcal{N}_{T}$, we have to add to $\operatorname{Mod}(\mathcal{K})$ all interpretations $\mathcal{J}$ that falsify Priest $\sqsubseteq$ Renter and that are minimally distant to some model $\mathcal{I}$ of $\mathcal{K}$, 
where distance is measured by one of the four measures defining the local semantics.

Let $\mathcal{I}$ be a model of $\mathcal{K}$. Then Priest ${ }^{\mathcal{I}} \subseteq$ Cleric $^{\mathcal{I}}$, Cleric $^{\mathcal{I}} \subseteq$ Renter $^{\mathcal{I}}$, and hence Priest ${ }^{\mathcal{I}} \subseteq$ Renter ${ }^{\mathcal{I}}$.

There are, in principle, two ways to minimally change $\mathcal{I}$ in such a way that Priest $\sqsubseteq$ Renter is no more satisfied. For one, we can add an individual $o \in \Delta \backslash \operatorname{Renter}^{\mathcal{I}}$ to Priest ${ }^{\mathcal{I}}$, provided Renter ${ }^{\mathcal{I}} \neq \Delta$, thus violating also Priest $\sqsubseteq$ Cleric. Alternatively, we can drop from Renter ${ }^{\mathcal{I}}$ an individual $o$ that is also in Priest ${ }^{\mathcal{I}}$, provided Priest ${ }^{\mathcal{I}} \neq \emptyset$, thus violating also Cleric $\sqsubseteq$ Renter.

Therefore, if $\mathcal{J}$ violates Priest $\sqsubseteq$ Renter and has minimal distance to $\mathcal{I}$ with respect to any of the four distances, we have

$$
\begin{aligned}
\text { - } \operatorname{dist}_{\subseteq}^{a}(\mathcal{I}, \mathcal{J}) & =\{\operatorname{Priest}(o)\} \text { or } \\
\operatorname{dist}_{\subseteq}^{a}(\mathcal{I}, \mathcal{J}) & =\{\operatorname{Renter}(o)\}, \text { for some } o \in \Delta ; \\
\text { - } \operatorname{dist}_{\#}^{a}(\mathcal{I}, \mathcal{J}) & =1 ; \\
\text { - } \operatorname{dist}_{\subseteq}^{s}(\mathcal{I}, \mathcal{J}) & =\{\text { Priest }\} \text { or } \text { dist }_{\subseteq}^{s}(\mathcal{I}, \mathcal{J})=\{\text { Renter }\} ; \\
\text { - } \text { dist }_{\#}^{s}(\mathcal{I}, \mathcal{J}) & =1 .
\end{aligned}
$$

Note that with respect to the symbol-based distances, minimal distance is also kept by adding more than one element to Priest or dropping more than one element from Renter.

We conclude that (1) any $\mathcal{J} \in \operatorname{Mod}($ Priest $\sqsubseteq$ Renter $)$ with minimal distance to $\mathcal{I}$ either satisfies Priest $\sqsubseteq$ Cleric or Cleric $\sqsubseteq$ Renter, (2) if Renter ${ }^{\mathcal{I}} \neq \Delta$, then there is a $\mathcal{J} \in \operatorname{Mod}($ Priest $\sqsubseteq$ Renter) with minimal distance to $\mathcal{I}$ such that $\mathcal{J}$ violates Priest $\sqsubseteq$ Cleric, and (3) if Priest ${ }^{\mathcal{I}} \neq \emptyset$, then there is a $\mathcal{J} \in \operatorname{Mod}$ (Priest $\sqsubseteq$ Renter) with minimal distance to $\mathcal{I}$ such that $\mathcal{J}$ violates Cleric $\sqsubseteq$ Renter. Thus,

$$
\operatorname{Mod}(\mathcal{K}) \cup \bigcup_{\mathcal{I} \in \operatorname{Mod}(\mathcal{K})} \underset{\mathcal{J} \in \operatorname{Mod}(\text { Priest } \sqsubseteq \text { Renter })}{\arg \min } \operatorname{dist}(\mathcal{I}, \mathcal{J})
$$

satisfies the conditions of Theorem 4.2 , which implies the claim for local semantics.

We next consider global semantics. As we have seen, the minimal distance between some $\mathcal{I} \in \operatorname{Mod}(\mathcal{K})$ and some $\mathcal{J} \in$ $\operatorname{Mod}($ Priest $\sqsubseteq$ Renter) is a set of cardinality one, or the number 1 . Moreover, for each such $\mathcal{I}$ there exist corresponding interpretations $\mathcal{J}$ with that minimal distance. If follows that for our example, contraction under a local semantics and its global counterpart coincide, that is, $\mathcal{K} \bullet_{\mathbb{G}_{z}^{y}} \mathcal{N}_{T}=\mathcal{K} \bullet_{\mathbb{L}_{z}^{y}} \mathcal{N}_{T}$. Thus, inexpressibility of contraction w.r.t. global semantics follows from the inexpressibility of contraction w.r.t. local semantics.

Observe that with a similar argument one can show that the expansion operator $\circ_{M^{\prime}}$ of Qi and Du [12] (and its stratified extension $\circ_{S}$ ), is not expressible in $D L$-Lite $\mathcal{F}_{\mathcal{R}}$. This operator is a variant of $\mathbb{G}_{\#}^{s}$ where in Equation (1) one considers only models $\mathcal{J} \in \operatorname{Mod}(\mathcal{N})$ that satisfy $A^{\mathcal{J}} \neq \emptyset$ for every $A$ occurring in $\mathcal{K} \cup \mathcal{N}$. The modification does not affect the inexpressibility, which can again be shown using Example 4.3. We also note that $\circ_{M^{\prime}}$ was developed for $\mathrm{KB}$ expansion with empty ABoxes and the inexpressibility comes from the non-empty ABox.
As we showed above, DL-Lite is closed neither under expansion nor under contraction. We investigate now whether the situation changes when we restrict evolution to affect only the ABox level of KBs.

\subsubsection{ABox Evolution}

We start with an example illustrating why ABox expansion w.r.t. $\mathbb{L}_{\subseteq}^{a}$ and $\mathbb{L}_{\#}^{a}$ is not expressible in DL-Lite $\mathcal{F} \mathcal{R}$.

Example 4.6. We turn again to our $\mathrm{KB} \mathcal{K}_{e x}$ and consider the scenario where we are informed that John is now a priest, formally $\mathcal{N}_{A}=\{\operatorname{Priest}($ john $)\}$. The TBox assertions essential for this example are EmpWife $\sqsubseteq$ Wife, Wife $\sqsubseteq$ $\exists$ HasHusband, $\exists$ HasHusband $\sqsubseteq$ Wife, and Priest $\sqsubseteq$ $\neg \exists$ HasHusband $^{-}$, while the essential ABox assertions are EmpWife(mary), HasHusband(mary, john), Priest(adam), and Priest(bob). Note also that every model of $\mathcal{K}_{e x}$ contains the atom Wife(mary). We show the inexpressibility of evolution w.r.t. $\mathbb{L}_{\subseteq}^{a}$ using Theorem 4.2 .

Under $\mathbb{L}_{\subseteq}^{a}$, in every $\mathcal{J} \in \mathcal{K} \circ \mathcal{N}_{A}$ one of four situations holds:

1. Mary is not a wife, that is, $\mathcal{J} \forall$ Wife(mary), and both Adam and Bob are priests, that is, $\mathcal{J} \models \operatorname{Priest(adam)}$ and $\mathcal{J} \models \operatorname{Priest}($ bob). Hence, $\mathcal{J} \models \operatorname{Priest(adam)~} \vee$ Priest(bob).

2. Mary has a husband, who is not John, say Sam. Due to minimality of change, both Adam and Bob are still priests, as in Case 1, and again $\mathcal{J} \models \operatorname{Priest(adam)~} \vee$ Priest(bob).

3. Mary is married to Adam, while Bob, due to mininality of change, is still a priest. That is, $\mathcal{J} \models \operatorname{Priest(adam)~} \vee$ Priest(bob). Moreover, the new husband cannot stay priest any longer and $\mathcal{J} \not \operatorname{Priest}$ (adam).

4. Mary is married to Bob and Adam remains a priest. Analogously to Case 3, we have $\mathcal{J} \models \operatorname{Priest(adam)~} \vee$ Priest(bob) and $\mathcal{J} \not$ Priest(bob).

In each situation we are in the conditions of Theorem 4.2 and therefore $\mathcal{K} \circ \mathcal{N}_{A}$ is not expressible in $D L$-Lite ${ }_{\mathcal{F} \mathcal{R}}$.

Next, we develop this example further so that it fits into all four local semantics and $\mathbb{G}^{a}$.

Theorem 4.7. DL-Lite $\mathcal{F}_{\mathcal{R}}$ is not closed under ABox expansion for $\mathbb{G}_{\subseteq}^{a}$ and $\mathbb{L}_{z}^{y}$, where $y \in\{s, a\}$, and $z \in\{\subseteq, \#\}$. Moreover, for local semantics this holds already when the initial $K B$ is written in DL-Lite core, and for $\mathbb{G}_{\subseteq}^{a}$ when the initial KB is writ-

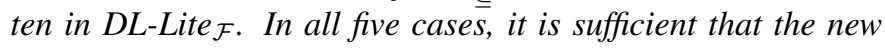
information consists of a single ABox axiom.

Proof. The inexpressibility of ABox expansion w.r.t. $\mathbb{L}_{\subseteq}^{a}$ has been shown in Example 4.6 . 
We turn now to expansion under $\mathbb{L}_{\#}^{a}$. We consider the following fragment of our running example:

$$
\begin{aligned}
\mathcal{T}=\{ & \text { EmpWife } \sqsubseteq \text { Wife, } \\
& \text { Wife } \sqsubseteq \exists \text { HasHusband, } \exists \text { HasHusband } \sqsubseteq \text { Wife }, \\
& \text { Priest } \left.\sqsubseteq \neg \exists \text { HasHusband }{ }^{-}\right\}, \\
\mathcal{A}=\{ & \text { EmpWife(mary), HasHusband(mary, john), } \\
& \text { Priest(adam), Priest(bob) }\} . \\
\mathcal{N}=\{ & \text { Priest(john) }\}, \\
\text { and } \mathcal{K}= & \mathcal{T} \cup \mathcal{A} .
\end{aligned}
$$

Let $\mathcal{I}$ be an arbitrary model of $\mathcal{K}$ and $\mathcal{J}$ a model of $\mathcal{N}$. Clearly, $\{$ Priest (john), HasHusband(mary, john) $\} \subset \mathcal{I} \ominus \mathcal{J}$. However, depending on $\mathcal{I}$, the symmetric difference $\mathcal{I} \ominus \mathcal{J}$ may contain further atoms. We distinguish between three main cases.

1. In the first case, Mary had more than one husband in $\mathcal{I}$. Then a minimally different $\mathcal{J} \in \operatorname{Mod}(\mathcal{N})$ is one where she is divorced from John, but stays married to the other husbands. Consequently, $\mathcal{I} \ominus \mathcal{J}$ contains no atoms other than the ones listed above, and the minimal distance between $\mathcal{I}$ and any $\mathcal{J}$ is $|\mathcal{I} \ominus \mathcal{J}|=2$.

2. In the second case, John was Mary's only husband in $\mathcal{I}$ and there was at least one individual other than John, say Sam, that was not a priest. Then a minimally different $\mathcal{J} \in \operatorname{Mod}(\mathcal{N})$ is one where Mary is divorced from John and marries such a Sam. Consequently, also HasHusband(mary, sam) $\in \mathcal{I} \ominus \mathcal{J}$, and the minimal distance between $\mathcal{I}$ and any $\mathcal{J}$ is $|\mathcal{I} \ominus \mathcal{J}|=3$. Note that for a $\mathcal{J}$ where Mary does not marry again, both atoms Wife(mary) and EmpWife(mary) have to be dropped so that $|\mathcal{I} \ominus \mathcal{J}|=4$, which is not minimal for $\mathcal{I}$.

3. In the third case, John was Mary's only husband in $\mathcal{I}$ and all individuals other than John were priests. Now, as in the previous case, for a $\mathcal{J}$ where Mary does not marry again, we have $|\mathcal{I} \ominus \mathcal{J}|=4$. Similarly, if Mary marries an individual $o \neq$ john that was a priest, then also $\{$ HasHusband(mary, $o$ ), Priest $(o)\} \subset \mathcal{I} \ominus \mathcal{J}$, so that $|\mathcal{I} \ominus \mathcal{J}|=4$.

We observe that in all three cases, including the subcases, one of Adam and Bob remains a priest in $\mathcal{J}$. In addition, for an $\mathcal{I}$ in the third case, it is possible that in a minimally different $\mathcal{J}$, Mary is married to one of Adam or Bob, hence, there is a $\mathcal{J}$ such that $\mathcal{J} \not=\operatorname{Priest}($ adam) and there is a $\mathcal{J}$ such that $\mathcal{J} \not$ Priest(bob). Again, we are in the case of Theorem 4.2, which proves that ABox expansion w.r.t. $\mathbb{L}_{\#}^{a}$ is not expressible in $D L$ Lite $_{\mathcal{F} \mathcal{R}}$.

To show the inexpressibility of symbol-based local semantics, we modify the $\mathrm{KB} \mathcal{K}=\mathcal{T} \cup \mathcal{A}$ introduced at the beginning of the proof, defining

$$
\begin{aligned}
& \mathcal{T}^{\prime}=\mathcal{T} \backslash\{\text { EmpWife } \sqsubseteq \text { Wife }\} \\
& \cup\left\{P_{0} \sqsubseteq \text { Priest }, P_{1} \sqsubseteq \text { Priest }, P_{2} \sqsubseteq \text { Priest }\right\} \\
&\left.\mathcal{A}^{\prime}=\mathcal{A} \backslash\{\text { EmpWife(mary })\right\} \\
& \cup\left\{P_{1}(\text { adam }), P_{2}(\text { bob })\right\}
\end{aligned}
$$

that is

$$
\begin{aligned}
\mathcal{T}^{\prime}=\{ & \text { Wife } \sqsubseteq \exists \text { HasHusband, } \\
& \exists \text { HasHusband } \sqsubseteq \text { Wife }, \\
& \text { Priest } \sqsubseteq \neg \exists \text { HasHusband }{ }^{-}, \\
& \left.P_{0} \sqsubseteq \text { Priest, } P_{1} \sqsubseteq \text { Priest, } P_{2} \sqsubseteq \text { Priest }\right\}, \\
\mathcal{A}^{\prime}=\{ & \text { HasHusband(mary, john }), \\
& \text { Priest (adam), } P_{1}(\text { adam }), \\
& \text { Priest (bob), } \left.P_{2} \text { (bob) }\right\},
\end{aligned}
$$

and $\mathcal{K}^{\prime}=\mathcal{T}^{\prime} \cup \mathcal{A}^{\prime}$. The new information $\mathcal{N}$ is as before. We want to show specifically that $\mathcal{K}^{\prime} \circ \mathcal{N}$ is not expressible in $D L$ Lite $_{\mathcal{F R}}$ both w.r.t. $\mathbb{L}_{\#}^{s}$ and w.r.t. $\mathbb{L}_{\#}^{s}$. We consider an aribtrary $\mathcal{I} \in \operatorname{Mod}\left(\mathcal{K}^{\prime}\right)$.

By a case analysis that is similar to the one in the proof for $\mathbb{L}_{\#}^{a}$, one can show that every $\mathcal{J}$ with minimal distance to $\mathcal{I}$ satisfies at least one of the assertions Priest(adam) and Priest(bob). Intuitively, the reason for this is that Priest(adam) cannot be removed from $\mathcal{I}$ without removing $P_{1}$ (adam), and Priest(bob) cannot be removed without removing $P_{2}$ (bob). Therefore, removing both atoms Priest(adam) and Priest(bob) leads to a distance between $\mathcal{I}$ and $\mathcal{J}$ that involves two additional symbols, namely $P_{1}$ and $P_{2}$, instead of only one additional symbol, namely either $P_{1}$ or $P_{2}$, involved in removing one of the two atoms.

Next, we exhibit models of $\mathcal{K}^{\prime} \circ \mathcal{N}$ that falsify one of the assertions Priest(adam) and Priest(bob). To this end, we consider a specific model $\mathcal{I}^{\prime}$ of $\mathcal{K}^{\prime}$. Let

$$
\begin{aligned}
\mathcal{I}^{\prime}=\{ & \text { Wife }(\text { mary }), \text { HasHusband }(\text { mary }, \text { john }), \\
& \text { Priest }(\text { adam }), P_{1}(\text { adam }), \\
& \text { Priest } \left.(\text { bob }), P_{2}(\text { bob })\right\} \\
\{ & \text { Priest } \left.(o), P_{0}(o) \mid o \in \Delta, o \notin\{\text { adam, bob, john }\}\right\} .
\end{aligned}
$$

One readily verifies that this is indeed a model of $\mathcal{K}^{\prime}$. We now check what the models $\mathcal{J}$ of $\mathcal{N}$ with minimal symbol-based distance to $\mathcal{I}^{\prime}$ look like. Clearly, $\mathcal{I}$ and $\mathcal{J}$ differ in that $\mathcal{J}$ misses the atom HasHusband(mary, john), but comprises the atom Priest(john). Hence, these two atoms are always elements of $\mathcal{I} \ominus \mathcal{J}$. Among the three cases we distinguished when analysing the example for $\mathbb{L}_{\#}^{a}$, the first two do not occur here, since Mary has only John as a husband and every individual, except John, is a priest. Therefore, the following situations are possible in such a $\mathcal{J}$ :

1. Mary does not remarry, which means that also the atom, Wife(mary) is in $\mathcal{I} \ominus \mathcal{J}$. Thus, in this case the set of symbols occurring in $\mathcal{I} \ominus \mathcal{J}$ is $\{$ HasHusband, Priest, Wife $\}$.

2. Mary marries someone other than Adam, Bob, or John, say Sam. Then, $\mathcal{I} \ominus \mathcal{J}$ contains also the three atoms $\left\{\right.$ Priest(sam), $P_{0}$ (sam), HasHusband(mary, sam) and the set of symbols occurring in $\mathcal{I} \ominus \mathcal{J}$ is $\left\{\right.$ HasHusband, Priest, $\left.P_{0}\right\}$.

3. Mary marries Adam. Then, $\mathcal{I} \ominus \mathcal{J}$ contains also the three atoms $\left\{\right.$ Priest(adam), $P_{1}$ (adam), HasHusband(mary, adam) $\}$ and the set of symbols occurring in $\mathcal{I} \ominus \mathcal{J}$ is $\left\{\right.$ HasHusband, Priest, $P_{1}$ \}.

4. Similarly, if Mary marries Bob, the set of symbols occurring in $\mathcal{I} \ominus \mathcal{J}$ is $\left\{\right.$ HasHusband, Priest, $P_{2}$ \}. 


\begin{tabular}{|c|c|c|c|c|}
\hline & \multicolumn{2}{|c|}{ Expansion } & \multicolumn{2}{|c|}{ Contraction } \\
\hline & TBox & ABox & TBox & ABox \\
\hline $\mathbb{L}_{\subset}^{a}$ & DL-Lite core $_{\text {e }}$ & DL-Lite core $_{\text {co }}$ & DL-Lite core $_{\text {ere }}$ & DL-Lite core $_{\text {ce }}$ \\
\hline $\mathbb{L}_{\#}^{\bar{a}}$ & DL-Lite core $_{\text {e }}$ & DL-Lite core & DL-Lite core $_{\text {ere }}$ & DL-Lite core $_{\text {co }}$ \\
\hline $\mathbb{L}_{C}^{s}$ & DL-Lite core $_{\text {e }}$ & DL-Lite core & DL-Lite core & DL-Lite core \\
\hline $\mathbb{L}_{\#}^{s}$ & DL-Lite core $_{\text {ce }}$ & DL-Lite $_{\text {core }}$ & DL-Lite core $_{\text {cre }}$ & DL-Lite core $_{\text {co }}$ \\
\hline $\mathbb{G}_{\subset}^{a}$ & DL-Lite ${ }_{\text {core }}$ & DL-Lite $_{\mathcal{F}}$ & DL-Lite $_{\text {core }}$ & DL-Lite $_{\mathcal{F}}$ \\
\hline $\mathbb{G}_{\#}^{a}$ & DL-Lite core & $?$ & DL-Lite core & $?$ \\
\hline $\mathbb{G}_{\subset}^{s}$ & DL-Lite core & $?$ & DL-Lite core $_{\text {ere }}$ & $?$ \\
\hline $\mathbb{G}_{\#}^{s}$ & DL-Lite core $_{\text {co }}$ & $?$ & DL-Lite core $_{\text {cre }}$ & $?$ \\
\hline
\end{tabular}

Table 1: Inexpressibility of KB evolution in $D L-$ Lite $_{\mathcal{F} \mathcal{R}}$. Each cell shows the smallest logic of the $D L$-Lite family in which evolution instances have been exhibited that are not expressible in $D L$-Lite $\mathcal{F} \mathcal{R}$.

Clearly, these four sets of symbols are minimal with respect to set inclusion, and since they all consist of three elements, they are also minimal with respect to cardinality. Two of them falsify one of the assertions Priest(adam) and Priest(bob). Together with the earlier observation that all $\mathcal{J} \in \mathcal{K}^{\prime} \circ \mathcal{N}$ satisfy Priest(adam) $\vee$ Priest(bob), this allows us to apply Theorem 4.2 and conclude the inexpressibility w.r.t. both $\mathbb{G}_{\subset}^{s}$ and $\mathbb{G}_{\#}^{s}$.

The inexpressibility of ABox expansion w.r.t. $\mathbb{G}_{\subset}^{a}$ can be shown similarly to the case of $\mathbb{L}_{\subset}^{a}$, but we need to add the assertion (funct HasHusband) to the TBox. In this way we ensure that in every model $\mathcal{I}$ of $\mathcal{K}$, Mary has only John as husband. To satisfy the assertion Wife(mary) she has to remarry, and the three options for obtaining a model $\mathcal{J}$ of $\mathcal{N},(1)$ marrying a nonpriest, (2) marrying an anonymous priest, and (3) marrying one of Adam or Bob, all lead to differences $\mathcal{I} \ominus \mathcal{J}$ that are minimal with regard to set inclusion. Again, application of Theorem 4.2 yields the claim.

\section{An anologous result holds for ABox contraction.}

Theorem 4.8. DL-Lite ${ }_{\mathcal{F} \mathcal{R}}$ is not closed under ABox contraction for $\mathbb{G}_{\subset}^{a}$ and $\mathbb{L}_{z}^{y}$, where $y \in\{s, a\}$, and $z \in\{\subseteq$, \#\}. Moreover, for local semantics this holds already when the initial $K B$ is written in $D L$-Lite core and for $\mathbb{G}_{\subseteq}^{a}$ when the initial KB is writ-

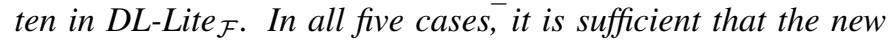
information consists of a single ABox axiom.

Proof. The proof of Theorem 4.7 can be almost literally adopted, if the original KB stays the same and the information to be contracted is the assertion HasHusband(mary, john).

Then, instead of concentrating on the models $\mathcal{J}$ of Priest(john) that are minimally different from models $\mathcal{I}$ of $\mathcal{K}$, we consider the set of models of $\mathcal{K}$, augmented by interpretations $\mathcal{J}$ that falsify HasHusband(mary, john) and are minimally different from models $\mathcal{I}$ of $\mathcal{K}$. We find that, for the semantics in question, each interpretation in the set considered satsifies Priest(adam) $\vee$ Priest(bob), while there is also a $\mathcal{J}_{a}$ that falsifies Priest(adam) and a $\mathcal{J}_{b}$ that falsifies Priest(bob). As before, Theorem 4.2 yields the claim.

In Table 1] we summarize our findings about the inexpressibility of $\mathrm{KB}$ evolution in $D L$-Lite FF $_{\mathcal{R}}$. The (in)expressibility of both, ABox expansion and contraction w.r.t. $\mathbb{G}_{\#}^{a}, \mathbb{G}_{\subseteq}^{s}$, and $\mathbb{G}_{\#}^{s}$ in $D L$-Lite F $_{\mathcal{R}}$ remains open problems.

\subsection{Conceptual Problems of MBAs}

We now discuss conceptual problems with all the local semantics. Recall Example 4.6 for local MBAs $\mathbb{L}_{\subset}^{a}$ and $\mathbb{L}_{\#}^{a}$. We note two problems. First, the divorce of Mary from John had a strange effect on the priests Bob and Adam. The semantics questions their celibacy and we have to drop the information that they are priests. This is counter-intuitive, since Mary and her divorce have nothing to do with any of these priests. Actually, the semantics also erases from the KB assertions about all other people belonging to concepts whose instances are not married, since potentially each of them is Mary's new husband. Second, a harmless clarification added to the TBox, namely that ministers are in fact clerics, strangely affects the whole class of clerics. The semantics of evolution "requires" one to allow marriages for clerics. This appears also strange, because intuitively the clarification on ministers does not contradict by any means the celibacy of clerics.

Also the four global MBAs have conceptual problems that were exhibited in Example 4.3. The restriction on rent subsidies that cuts the payments for wives introduces a counterintuitive choice for employed wives. Under the symbol-based global semantics, they must either collectively get rid of their husbands or collectively lose the subsidy. Under atom-based semantics the choice is an individual one.

Summing up on both global and local MBAs, they focus on minimal change of models of $\mathrm{KBs}$ and, hence, introduce choices that cannot be captured in DL-Lite, which owes its good computational properties to the absence of disjunction. This mismatch with regard to the structural properties of KBs leads to counterintuitive and undesired results, like inexpressibility in DL-Lite and erasure of the entire KB. Therefore, we claim that these semantics are not suitable for the evolution of $D L$ Lite KBs and now study evolution according to formula-based approaches.

\section{Formula-based Approaches to KB Evolution}

Under formula-based approaches, the objects of change are sets of formulae. We recall that without loss of generality we can consider only closed KBs, that is, if $\mathcal{K} \models \alpha$ for some $D L$ Lite $_{\mathcal{F} \mathcal{R}}$ assertion $\alpha$, then $\alpha \in \mathcal{K}$.

\subsection{Classical Formula-based Approaches}

Given a closed $\mathrm{KB} \mathcal{K}$ and new knowledge $\mathcal{N}$, a natural way to define the result of expansion seems to choose a maximal subset $\mathcal{K}_{m}$ of $\mathcal{K}$ such that $\mathcal{K}_{m} \cup \mathcal{N}$ is coherent and to define $\mathcal{K} \circ \mathcal{N}$ as $\mathcal{K}_{m} \cup \mathcal{N}$. However, a problem here is that in general such a $\mathcal{K}_{m}$ is not unique.

Let $\mathcal{M}_{e}(\mathcal{K}, \mathcal{N})$ be the set of all such maximal $\mathcal{K}_{m}$. In the past, several approaches to combine all elements of $\mathcal{M}_{e}(\mathcal{K}, \mathcal{N})$ into one set of formulae, which is then added to $\mathcal{N}$, have been proposed [9, 10]. The two main ones are known as CrossProduct, or CP for short, and When In Doubt Throw It Out, 
or WIDTIO for short. The corresponding sets $\mathcal{K}_{C P}$ and $\mathcal{K}_{\text {WIDTIO }}$ are defined as follows:

$$
\begin{aligned}
\mathcal{K} \circ^{\circ P} \mathcal{N} & =\mathcal{N} \cup\left\{\bigvee_{\mathcal{K}_{m} \in \mathcal{M}_{e}(\mathcal{K}, \mathcal{N})}\left(\bigwedge_{\varphi \in \mathcal{K}_{m}} \varphi\right)\right\}, \\
\mathcal{K}{ }^{\circ}{ }_{\text {WIDTIO }} \mathcal{N} & =\mathcal{N} \cup\left(\bigcap_{\mathcal{K}_{m} \in \mathcal{M}_{e}(\mathcal{K}, \mathcal{N})} \mathcal{K}_{m}\right) .
\end{aligned}
$$

In $C P$ one adds to $\mathcal{N}$ the disjunction of all $\mathcal{K}_{m}$, viewing each $\mathcal{K}_{m}$ as the conjunction of its assertions, while in WIDTIO one adds to $\mathcal{N}$ those formulas present in all $\mathcal{K}_{m}$. In terms of models, every model of $\mathcal{K}_{\text {WIDTIO }}$ is also a model of $\mathcal{K}_{C P}$, whose models in turn are exactly the interpretations satisfying some of the $\mathcal{K}_{m}$.

We can naturally extend this approach to the case of contraction. Indeed, let $\mathcal{K}_{m}$ be a maximal subset of $\mathcal{K}$ such that $\mathcal{K}_{m} \not \models \alpha$ for each $\alpha \in \mathcal{N}$ and let $\mathcal{M}_{c}(\mathcal{K}, \mathcal{N})$ be the set of all such maximal $\mathcal{K}_{m}$. Then we can define contraction under $C P$ and WIDTIO as follows:

$$
\begin{aligned}
\mathcal{K} \bullet \bullet_{C P} \mathcal{N} & =\left\{\bigvee_{\mathcal{K}_{m} \in \mathcal{M}_{c}(\mathcal{K}, \mathcal{N})}\left(\bigwedge_{\varphi \in \mathcal{K}_{m}} \varphi\right)\right\}, \\
\mathcal{K} \bullet_{\text {WIDTIO }} \mathcal{N} & =\bigcap_{\mathcal{K}_{m} \in \mathcal{M}_{c}(\mathcal{K}, \mathcal{N})} \mathcal{K}_{m} .
\end{aligned}
$$

Next, we show that these semantics satisfy the evolution postulates defined in Section 3.2 .

Proposition 5.1. Expansion (resp., contraction) of a DLLite $_{\mathcal{F}} K B$ under operator $\circ_{X}\left(\right.$ resp. $\left.\bullet_{X}\right)$, where $X \in$ $\{C P, W I D T I O\}$, satisfies $\boldsymbol{E 1 - E 5 ~ ( r e s p . ~ C 1 - C 3 ~ a n d ~ C 5 ) . ~ H o w - ~}$ ever, contraction under both $C P$ and WIDTIO does not satisfy $\boldsymbol{C 4}$.

Proof. The claim for E1, E2, E5, C1, C2, and C5 follows directly from the definitions of the operators. E3 (resp., C3) follows from the observation that if $\mathcal{K} \models \mathcal{N}$, (resp., if $\mathcal{K} \not \alpha$ for each $\alpha \in \mathcal{N})$, then $\mathcal{M}_{e}(\mathcal{K}, \mathcal{N})=\{\mathcal{K}\}\left(\operatorname{resp} ., \mathcal{M}_{c}(\mathcal{K}, \mathcal{N})=\right.$ $\{\mathcal{K}\}$ ). Finally, $\mathbf{E 4}$ follows from the following observation:

$C P$ : Assume that $\mathcal{J}$ is a model of $\left(\mathcal{K}^{\circ}{ }_{C P} \mathcal{N}_{1}\right) \cup \mathcal{N}_{2}$. Then $\mathcal{J}=$ $\mathcal{N}_{1}, \mathcal{J} \models \mathcal{N}_{2}$, and $\mathcal{J} \models \mathcal{K}_{m}^{\prime}$ for some $\mathcal{K}_{m}^{\prime} \in \mathcal{M}_{e}\left(\mathcal{K}, \mathcal{N}_{1}\right)$. But in this case we have that $\mathcal{K}_{m}^{\prime} \cup \mathcal{N}_{1} \cup \mathcal{N}_{2}$ is satisfiable and therefore $\mathcal{K}_{m}^{\prime} \in \mathcal{M}_{e}\left(\mathcal{K}, \mathcal{N}_{1} \cup \mathcal{N}_{2}\right)$, which shows the claim.

WIDTIO: First observe that for each $\mathcal{K}_{m}^{\prime \prime} \in \mathcal{M}_{e}\left(\mathcal{K}, \mathcal{N}_{1} \cup\right.$ $\left.\mathcal{N}_{2}\right)$ there exists $\mathcal{K}_{m}^{\prime} \in \mathcal{M}_{e}\left(\mathcal{K}, \mathcal{N}_{1}\right)$ such that $\mathcal{K}_{m}^{\prime \prime} \subseteq$ $\mathcal{K}_{m}^{\prime}$. Assume that $\mathcal{J}$ is a model of $\left(\mathcal{K} \circ_{\text {WIDTIO }} \mathcal{N}_{1}\right) \cup$ $\mathcal{N}_{2}$. Then $\mathcal{J} \models \mathcal{N}_{1}, \mathcal{J} \models \mathcal{N}_{2}$, and $\mathcal{J} \models \alpha$ for each $\alpha \in \bigcap_{\mathcal{K}_{m}^{\prime} \in \mathcal{M}_{e}\left(\mathcal{K}, \mathcal{N}_{1}\right)} \mathcal{K}_{m}^{\prime}$. Due to the obser-

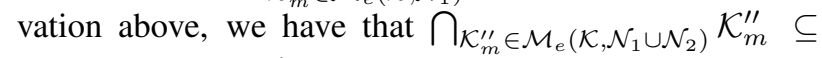
$\bigcap_{\mathcal{K}_{m}^{\prime} \in \mathcal{M}_{e}\left(\mathcal{K}, \mathcal{N}_{1}\right)} \mathcal{K}_{m}^{\prime}$, which shows the claim.

To see that contraction under both $C P$ and WIDTIO does not satisfy $\mathbf{C 4}$, consider the following example. Let $\mathcal{K}$ consist of a TBox $\{A \sqsubseteq B\}$ and an ABox $\{A(a)\}$, and let $\mathcal{N}$ consist of an assertion $B(a)$. It is easy to see that $\mathcal{M}_{c}(\mathcal{K}, \mathcal{N})=\left\{\mathcal{K}_{m}^{1}, \mathcal{K}_{m}^{2}\right\}$, where $\mathcal{K}_{m}^{1}=\{A(a)\}$ and $\mathcal{K}_{m}^{2}=$
$\{A \sqsubseteq B)\}$. Then observe that the interpretation $\mathcal{J}=$ $\{B(a)\}$ is a model of $\left(\mathcal{K} \bullet{ }_{C P} \mathcal{N}\right) \cup \mathcal{N}$ since it is a model of $\mathcal{K}_{m}^{2} \cup \mathcal{N}$, and it is a model of $\left(\mathcal{K} \bullet_{\text {WIDTIO }} \mathcal{N}\right) \cup \mathcal{N}$ since $\mathcal{K} \bullet$ WIDTIO $\mathcal{N}=\emptyset$. This concludes the proof.

Intuitively, contraction under the two operators does not satisfy $\mathbf{C 4}$, since we restrict ourselves to $D L$-Lite $_{\mathcal{F} \mathcal{R}}$, and therefore, when getting rid of the information in $\mathcal{N}$, we are not able to be too precise and delete only what is really required, but we have to delete too much information.

Next, we observe some built-in shortcomings of the two semantics. Consider the following example.

Example 5.2. We consider again our running example. Suppose we obtain the new information that priests no longer obtain rental subsidies. This can be captured by the set of TBox assertions $\mathcal{N}_{T}=$ Priest $\sqsubseteq \neg$ Renter $\}$. We now incorporate this information into our $\mathrm{KB}$, under both $C P$ and $W I D$ TIO semantics. Clearly, $\mathcal{K}_{e x} \cup \mathcal{N}_{T}$ is not coherent and to resolve the conflict one can drop either Priest $\sqsubseteq$ Cleric or Cleric $\sqsubseteq$ Renter. Hence, $\mathcal{M}_{e}\left(\mathcal{K}_{e x}, \mathcal{N}_{T}\right)=\left\{\mathcal{K}_{m}^{(1)}, \mathcal{K}_{m}^{(2)}\right\}$, where $\mathcal{K}_{m}^{(1)}=\mathcal{K}_{e x} \backslash\{$ Priest $\sqsubseteq$ Cleric $\}$, and $\mathcal{K}_{m}^{(2)}=$ $\mathcal{K}_{e x} \backslash\{$ Cleric $\sqsubseteq$ Renter $\}$. Consequently, the results of evolving $\mathcal{K}$ with respect to $\mathcal{N}_{T}$ under the two semantics are

$$
\begin{aligned}
& \mathcal{K}_{\text {ex }}{ }^{\circ}{ }_{C P} \mathcal{N}_{T}=\mathcal{N}_{T} \cup((\mathcal{K} \backslash\{\text { Priest } \sqsubseteq \text { Cleric }\}) \\
&\vee(\mathcal{K} \backslash\{\text { Cleric } \sqsubseteq \text { Renter }\})) \\
& \mathcal{K}_{e x} \circ_{\text {WIDTIO }} \mathcal{N}_{T}=\mathcal{N}_{T} \cup\left(\mathcal{K}_{m}^{(1)} \cap \mathcal{K}_{m}^{(2)}\right) \\
&=\left(\mathcal{N}_{T} \cup \mathcal{K}_{e x}\right) \backslash\{\text { Priest } \sqsubseteq \text { Cleric }, \\
&\text { Cleric } \sqsubseteq \text { Renter }\},
\end{aligned}
$$

where in the first formula we have combined DL notation with first order logic notation.

Intuitively, $C P$ does not lose information, but the price to pay is that the resulting $\mathrm{KB}$ can be exponentially larger than the original $\mathrm{KB}$, since there can exist exponentially many $\mathcal{K}_{m}$. Indeed, consider a KB that contains for each $i \in\{1, \ldots, n\}$, three concepts $A_{i}, B_{i}, C_{i}$ and the two inclusion assertions $A_{i} \sqsubseteq B_{i}$ and $B_{i} \sqsubseteq C_{i}$; and the new information that states that $A_{i}$ and $C_{i}$ are disjoint for all $i$. Then, there are $2^{n}$ many maximal coherent subsets $\mathcal{K}_{m}$. In addition, as Example 5.2 shows, even if $\mathcal{K}$ is a $D L$-Lite $_{\mathcal{F} \mathcal{R}} \mathrm{KB}$, the result may not be representable in $D L$-Lite $_{\mathcal{F} \mathcal{R}}$ any more since it requires disjunction. This effect is also present if the new knowledge involves only ABox assertions.

WIDTIO, on the other extreme, is expressible in DL-Lite. However, it can lose many assertions, which may be more than one is prepared to tolerate. Even, if one deems this loss acceptable, one has to cope with the fact that it is computationally complex to decide whether an assertion belongs to $\mathcal{K}{ }^{\text {WIDTIO }} \mathcal{N}$. This problem is already difficult if our KBs are TBoxes that are specified in the simplest variant of DL-Lite. We note that the following theorem can be seen as a sharpening of a result about WIDTIO for propositional Horn theories in [10], obtained with a different reduction than ours. 
Theorem 5.3. For a $D L$-Lite ${ }_{\mathcal{F}} \mathcal{R} B \mathcal{K}$ and new information $\mathcal{N}$, deciding whether an assertion is in $\mathcal{K} \circ_{\text {WIDTIO } \mathcal{N}}$ is coNPcomplete. Moreover, hardness holds already for DL-Lite core KBs with empty ABoxes.

Proof. The membership in coNP is straightforward: to check that an assertion $\varphi$ is not in $\mathcal{K}{ }^{\circ}$ WIDTIO $\mathcal{N}$, guess a $\mathcal{K}_{m}$ from $\mathcal{M}_{e}(\mathcal{K}, \mathcal{N})$ and verify that $\mathcal{K}_{m} \cup \mathcal{N} \not \models \varphi$. To see that this is in fact a non-deterministic polynomial time procedure, note that a subset $\mathcal{K}^{\prime}$ of $\mathcal{K}$ is an element of $\mathcal{M}_{e}(\mathcal{K}, \mathcal{N})$ if for any formula $\gamma \in \mathcal{K} \backslash \mathcal{K}^{\prime}$, we have that $\mathcal{K}^{\prime} \cup \mathcal{N} \cup\{\gamma\}$ is not coherent. This can be verified in polynomial time for $D L$-Lite KBs.

That the expansion problem is coNP-hard is shown by a reduction of 3SAT, which is illustrated in Figure 3 . Let $\psi$ be a 3-CNF formula. Our plan is to construct $\mathrm{KBs} \mathcal{K}^{\psi}$ and $\mathcal{N}^{\psi}$, both consisting only of inclusion assertions. We single out one assertion $\varphi$ of $\mathcal{K}^{\psi}$ such that $\psi$ is unsatisfiable if and only if $\mathcal{K} \circ_{\text {WIDTIO }} \mathcal{N}=\varphi$.

Let $p_{1}, \ldots, p_{r}$ be the propositional variables occurring in $\psi$. Without loss of generality, we can assume that each $p_{l}$ occurs both positively and negatively in $\psi$. Suppose $\psi$ is a conjunction $\psi_{1} \wedge \cdots \wedge \psi_{n}$ of $n$ clauses. Each clause $\psi_{i}$ is a disjunction of three literals $L_{i 1} \vee L_{i 2} \vee L_{i 3}$, where either $L_{i j}=p_{i}$ for some variable $p_{i}$, in which case we say that $L_{i j}$ is positive, or $L_{i j}=\neg p_{i}$, in which case we say that $L_{i j}$ is negative.

The KB $\mathcal{K}^{\psi}$ models the clauses and their literals by a set of concept inclusion assertions. For each literal $L_{i j}$ we introduce two concepts $X_{i j}$ and $Y_{i j}$, together with the assertion

$$
X_{i j} \sqsubseteq Y_{i j}, \quad \text { for } i \in\{1, \ldots, n\}, j \in\{1,2,3\} .
$$

The KB $\mathcal{K}^{\psi}$ contains these inclusions and the disjointness axiom

$$
\varphi=Z_{0} \sqsubseteq \neg Z_{n} .
$$

The new KB $\mathcal{N}^{\psi}$ consists of two parts, one that models the possible truth values of the literals, and a second that models the logical connections of the literals.

To model the values assigned to the literals by a truth value assignment, we introduce for each propositional variable $p_{l}$ three concepts $S_{l}, P_{l}$, and $N_{l}$. We insert into $\mathcal{N}^{\psi}$ the inclusion

$$
S_{l} \sqsubseteq X_{i j} \quad \text { whenever } p_{l} \text { is the variable of } L_{i j} \text {. }
$$

We connect the corresponding concepts $Y_{i j}$ to either $P_{l}$ or $N_{l}$, depending on whether $p_{l}$ occurs positively or negatively in $L_{i j}$. More precisely, we add to $\mathcal{N}^{\psi}$ the inclusion

$$
\begin{array}{cl}
Y_{i j} \sqsubseteq P_{l}, & \text { if } L_{i j}=p_{l}, \\
Y_{i j} \sqsubseteq N_{l}, & \text { if } L_{i j}=\neg p_{l} .
\end{array}
$$

Finally, we add to $\mathcal{N}^{\psi}$ the disjointness axiom $P_{l} \sqsubseteq \neg N_{l}$.

The intuition behind the reduction becomes clear if we view each inclusion axiom as an arc in a directed graph, whose nodes are the concepts. By construction, since $p_{l}$ occurs both positively and negatively in $\psi$, there are is a path in $\mathcal{K}^{\psi} \cup \mathcal{N}^{\psi}$ from $S_{l}$ to $P_{l}$ and another one from $S_{l}$ to $N_{l}$. Since in any model of $\mathcal{K}^{\psi} \cup \mathcal{N}^{\psi}$, the concepts $P_{l}$ and $N_{l}$ are disjoint, the concept
$S_{l}$, which is contained in both, is interpreted as the empty set, which makes the KB incoherent. This can only be prevented by dropping either all paths from $S_{l}$ to $N_{l}$ or all paths from $S_{l}$ to $P_{l}$. Keeping in a maximal coherent subset $\mathcal{K}_{m} \subseteq \mathcal{K}^{\psi}$ all the paths from $S_{l}$ to $P_{l}$, corresponds to assigning to $p_{l}$ the value true. Keeping only the paths from $S_{l}$ to $P_{l}$, corresponds to assigning to $p_{l}$ the value false.

To model the logic of the clauses, we introduce into $\mathcal{N}^{\psi}$ six inclusion axioms per clause. To this end, we use, in addition to $Z_{0}$ and $Z_{n}$, another $n-1$ concepts $Z_{1}, \ldots, Z_{n-1}$. Then, the six inclusions for the $i$-th clause are

$$
\begin{aligned}
Z_{i-1} & \sqsubseteq X_{i j} \\
Y_{i j} & \sqsubseteq Z_{i}
\end{aligned} \quad \text { for } j \in\{1,2,3\} .
$$

Under the graph view of the $\mathrm{KB} \mathcal{K}^{\psi} \cup \mathcal{N}^{\psi}$, one can walk from $Z_{i-1}$ to $Z_{i}$ only along three possible paths, passing one of the arcs $X_{i j} \sqsubseteq Y_{i j}$ corresponding to the literals $L_{i j}, j \in\{1,2,3\}$. This models the disjunction of the three literals appearing in the $i$-th clause $\psi_{i}$. To walk from $Z_{0}$ to $Z_{n}$, one has to take all the $n$ steps, from $Z_{i-1}$ to $Z_{i}$, for $i \in\{1, \ldots, n\}$. This models the conjunction of the $n$ clauses in $\psi$. A path from $Z_{0}$ to $Z_{n}$ forces $Z_{0}$ to be a subset of $Z_{n}$ in every model of the path. Together with the disjointness axiom $\varphi=\left(Z_{0} \sqsubseteq \neg Z_{n}\right)$, this implies that $Z_{0}$ is empty, which is not possible, if we want our $\mathrm{KB}$ to be coherent.

We are now in a position to show that $\varphi$ does not follow from $\mathcal{K}^{\psi}{ }^{\circ}{ }_{\text {WIDTIO }} \mathcal{N}^{\psi}$ if and only if $\psi$ is satisfiable. To this end, assume that $\psi$ is satisfiable and let $\alpha$ be a satisfying assignment. Let $\mathcal{K}^{\prime} \subseteq \mathcal{K}^{\psi}$ contain $X_{i j} \sqsubseteq Y_{i j}$ if and only if $\alpha\left(L_{i j}\right)=$ true. Suppose, $\alpha$ satisfies the $j$-th literal of the $i$-th clause, $L_{i j}$. Then $\mathcal{K}^{\prime} \cup \mathcal{N}^{\psi}$ contains a path from $Z_{i-1}$ to $Z_{i}$, passing through $X_{i j}$ and $Y_{i j}$. Since, by assumption, $\alpha$ satisfies every clause in $\psi$, the $\mathrm{KB} \mathcal{K}^{\prime} \cup \mathcal{N}^{\psi}$ contains a path from $Z_{0}$ to $Z_{n}$. As seen above, adding $\varphi$ to $\mathcal{K}^{\prime} \subseteq \mathcal{N}^{\psi}$ would lead to an incoherent KB. Thus, with $\mathcal{K}^{\prime}$ we have exhibited an element of $\mathcal{M}_{e}\left(\mathcal{K}^{\psi}, \mathcal{N}^{\psi}\right)$ that does not contain $\varphi$, so that $\varphi$ is not in the intersection of the elements of $\mathcal{M}_{e}\left(\mathcal{K}^{\psi}, \mathcal{N}^{\psi}\right)$ and thereofore does not follow from $\mathcal{K}^{\psi}$ o $_{\text {WIDTIO }} \mathcal{N}^{\psi}$.

Next, assume that $\psi$ is unsatisfiable, and let $\mathcal{K}_{m}$ be a maximal subset of $\mathcal{K}^{\psi}$ such that $\mathcal{N} \cup \mathcal{K}_{m}$ is coherent. Let $\alpha$ be the assignment such that $\alpha\left(p_{l}\right)=$ true if $\left(X_{i j} \sqsubseteq Y_{i j}\right) \in \mathcal{K}_{m}$ for some positive literal $L_{i j}=p_{l}$, and $\alpha\left(p_{l}\right)=$ false otherwise. This assignment, like all assignments, by assumption falsifies $\psi$ and in particular falsifies one clause, say the $i$-th one. Then all literals of that clause are falsified by $\alpha$.

Consider a literal of that clause, say $L_{i j}$. We make a case analysis as to wheter $L_{i j}$ is a positive or a negative literal. Suppose $L_{i j}$ is positive, say $L_{i j}=p_{l}$. Then $\alpha\left(p_{l}\right)=$ false, which means that the condition for $\alpha\left(p_{l}\right)$ being true true does not hold. Then $\mathcal{K}_{m}$ contains no inclusion corresponding to a positive $p_{l}$-literal. In particular, the inclusion $X_{i j} \sqsubseteq Y_{i j}$ for $L_{i j}$ is not in $\mathcal{K}_{m}$. Suppose $L_{i j}$ is positive, say $L_{i j}=\neg p_{l}$. Then $\alpha\left(p_{l}\right)=$ true. By definition of $\alpha$, some inclusion corresponding to a positive $p_{l}$-literal is present in $\mathcal{K}_{m}$. Hence, no arc for a negative $p_{l}$-literal is in $\mathcal{K}_{m}$, because otherwise $S_{l}$ would be incoherent. Therefore, the inclusion $X_{i j} \sqsubseteq Y_{i j}$ corresponding to $L_{i j}$ is not in $\mathcal{K}_{m}$. 

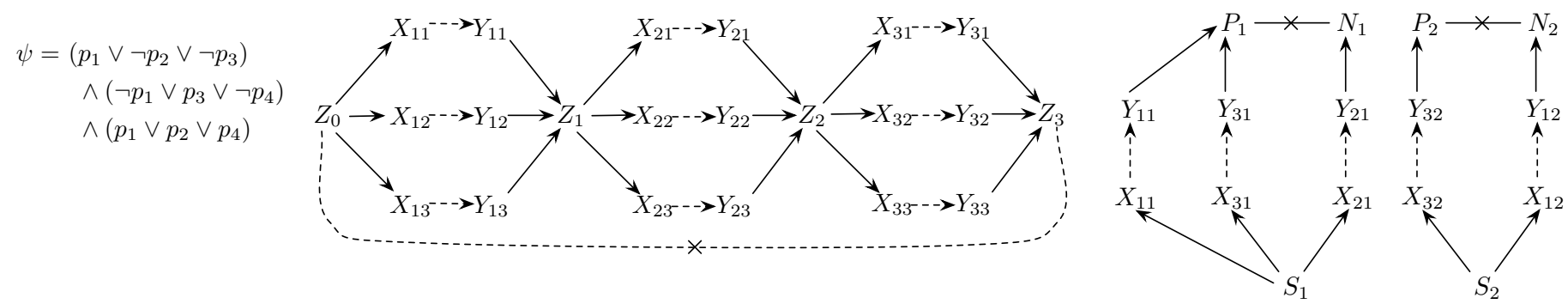

Figure 3: Illustration of the 3SAT reduction.

In summary, we have seen that there is no path from $Z_{i-1}$ to $Z_{i}$ in $\mathcal{K}_{m} \cup \mathcal{N}^{\psi}$. Consequently, $Z_{0} \sqsubseteq Z_{n}$ does not follow from $\mathcal{K}_{m} \cup \mathcal{N}^{\varphi}$, so that $\varphi=\left(Z_{0} \sqsubseteq \neg Z_{n}\right)$ is in $\mathcal{K}_{m}$, due to the maximality of $\mathcal{K}_{m}$. Since $\mathcal{K}_{m}$ was arbitrary, $\mathcal{K}^{\psi}{ }_{{ }{ }_{\text {WIDTIO }}} \mathcal{N}^{\psi} \models$ $\varphi$.

This shows that $\mathcal{K}^{\psi}{ }^{\circ}$ WIDTIO $\mathcal{N}^{\psi}=\varphi$ if and only if $\psi$ is unsatisfiable, which completes the proof.

Thus, both $C P$ and WIDTIO semantics are computationally problematic, even for languages such as $D L$-Lite $_{\mathcal{F} \mathcal{R}}$, where the closure of a $\mathrm{KB}$ is always finite. Therefore, we conclude that neither $C P$ nor $W I D T I O$ is proper for practical solutions. In the following, we introduce a semantics that can help to overcome the issue of intractability.

\subsection{Bold Semantics}

As we have seen above, the classical approaches $C P$ and WIDTIO may pose practical challenges in the case of $D L$ Lite $_{\mathcal{F} \mathcal{R}}$. Indeed, the former one is inexpressible in $D L$-Lite $\mathcal{F}_{\mathcal{R}}$, since it requires disjunction, but even for more expressive languages where $C P$ is expressible, the resulting $\mathrm{KB}$, after a series of evolutions, is going to be very complicated and overloaded. The latter semantics is always expressible in DL-Lite $\mathcal{F}_{\mathcal{R}}$; computing the result under it, however, is computationally hard even

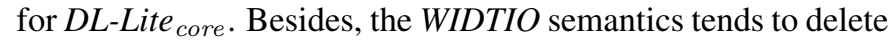
too much information.

Recall that both $C P$ and WIDTIO semantics were proposed to combine all elements of $\mathcal{M}_{e}(\mathcal{K}, \mathcal{N})$ or $\mathcal{M}_{c}(\mathcal{K}, \mathcal{N})$ into a single $\mathrm{KB}$. We propose another way to deal with the problem of multiple maximal KBs: instead of combining the different $\mathcal{K}_{m}$, we suggest to choose one of them. We call this semantics bold. More formally, we say that $\mathcal{K}^{\prime}$ is a result of expansion (resp., contraction) of $\mathcal{K}$ w.r.t. $\mathcal{N}$ if $\mathcal{K}^{\prime} \equiv \mathcal{K}_{m} \cup \mathcal{N}$ for some $\mathcal{K}_{m} \in$ $\mathcal{M}_{e}(\mathcal{K}, \mathcal{N})$ (resp., $\mathcal{K}^{\prime} \equiv \mathcal{K}_{m}$ for some $\mathcal{K}_{m} \in \mathcal{M}_{c}(\mathcal{K}, \mathcal{N})$ ). An obvious drawback of this approach is that the choice of $\mathcal{K}_{m}$ is not deterministic. Consider the following example.

Example 5.4. Consider the $\mathrm{KB}$ and the new information from Example 5.2. As shown there, $\mathcal{M}\left(\mathcal{K}_{\text {ex }}, \mathcal{N}_{T}\right)=\left\{\mathcal{K}_{m}^{(1)}, \mathcal{K}_{m}^{(2)}\right\}$. According to bold semantics the result of expansion is a $\mathrm{KB}$ $\mathcal{K}^{\prime}=\mathcal{N} \cup \mathcal{K}_{m}$ for some $\mathcal{K}_{m} \in \mathcal{M}\left(\mathcal{K}_{e x}, \mathcal{N}_{T}\right)$. Thus, the result of expansion is either $\mathcal{N}_{T} \cup \mathcal{K}_{e x} \backslash$ Priest $\sqsubseteq$ Cleric $\}$ or $\mathcal{N}_{T} \cup \mathcal{K}_{\text {ex }} \backslash\{$ Cleric $\sqsubseteq$ Renter $\}$.

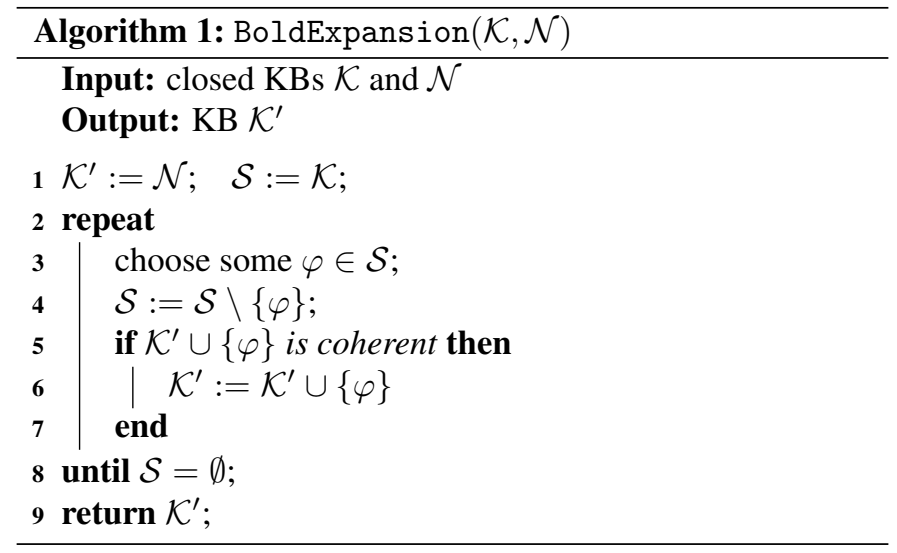

We continue now with a check of how bold semantics satisfies the evolution postulates. But first observe that the postulates E4, E5, and $\mathbf{C 5}$ do not make much sense in the context of bold semantics, due to its non-determinism. Therefore, we first propose an alternative version of those postulates to take into consideration the non-determinism of bold semantics:

E4B: For each $\mathcal{K}_{m}^{\prime \prime} \in \mathcal{M}_{e}\left(\mathcal{K}, \mathcal{N}_{1 e} \cup \mathcal{N}_{2 e}\right)$, there exists a $\mathcal{K}_{m}^{\prime} \in \mathcal{M}_{e}\left(\mathcal{K}, \mathcal{N}_{1 e}\right)$ such that $\mathcal{K}_{m}^{\prime} \models \mathcal{K}_{m}^{\prime \prime}$.

E5B: Expansion should not depend on the syntactical representation of knowledge, that is, if $\mathcal{K}_{1} \equiv \mathcal{K}_{2}$ and $\mathcal{N}_{1 e} \equiv \mathcal{N}_{2 e}$, then $\mathcal{M}_{e}\left(\mathcal{K}_{1}, \mathcal{N}_{1 e}\right) \equiv \mathcal{M}_{e}\left(\mathcal{K}_{2}, \mathcal{N}_{2 e}\right)$.

C5B: Contraction should not depend on the syntactical representation of knowledge, that is, if $\mathcal{K}_{1} \equiv \mathcal{K}_{2}$ and $\mathcal{N}_{1 c} \equiv \mathcal{N}_{2 c}$, then $\mathcal{M}_{c}\left(\mathcal{K}_{1}, \mathcal{N}_{1 c}\right) \equiv$ $\mathcal{M}_{c}\left(\mathcal{K}_{2}, \mathcal{N}_{2 c}\right)$.

Proposition 5.5. For the evolution of DL-Lite $\mathcal{F}_{\mathcal{R}} K B s$ under bold semantics the following holds:

- Expansion satisfies E1-E3, E4B, and E5B;

- Contraction satisfies C1-C3 and C5B, but not C4.

Proof. The claim for E1, E2, E5B, C1, C2, and C5B follows directly from the definitions of the operators. The claim for $\mathbf{E 3}$, E4B, and $\mathbf{C 3}$ and the fact that contraction does not satisfy $\mathbf{C 4}$ can be proved similarly to the corresponding claims in Proposition 5.1 


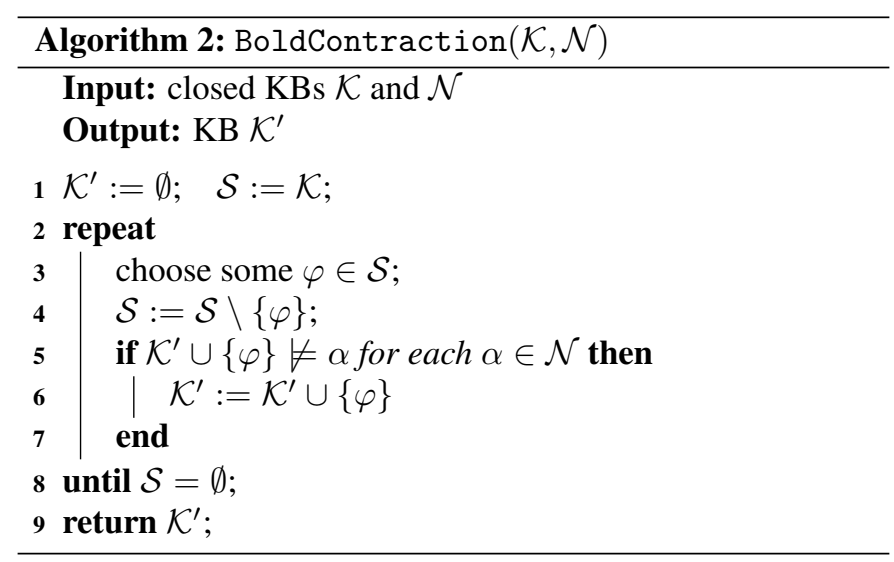

Which of the two possible results in Example 5.4 should one choose? We claim that the choice is domain-dependent and, consequently, it should be made by a user/domain expert. In our particular example, the right choice seems to pick the second $\mathrm{KB}$ since it is possible that clerics do not receive rent subsidies, while the first option where priests stop being clerics does not make sense.

\subsubsection{Bold Semantics without User Preferences}

Consider the case when the user does not have any preferences and any of the possible results of evolution would be satisfactory. In this case, choosing an arbitrary $\mathcal{K}_{m}$ has the advantage that the result of evolution can be computed in polynomial time. Algorithms 1 and 2 can be used to compute the result of expansion or contraction, respectively, in a non-deterministic manner.

Theorem 5.6. For DL-Lite $\mathcal{F}_{\mathcal{R}} K B s \mathcal{K}$ and $\mathcal{N}$, the algorithms BoldExpansion and BoldContraction run in time polynomial in $|\mathcal{K} \cup \mathcal{N}|$ and compute a bold expansion and a bold contraction of $\mathcal{K}$ by $\mathcal{N}$, respectively.

Proof. The fact that the algorithms compute the results of expansion and contraction, respectively, is obvious. To prove polynomiality, observe that the algorithms loop as many times as there are assertions in $\mathcal{K}$. The crucial steps are the coherence steps for BoldExpansion and the entailment checks for BoldContraction. It is well known, however, that in $D L$ Lite $_{\mathcal{F} \mathcal{R}}$ these checks can be done in polynomial time.

\subsubsection{Bold Semantics with User Preferences}

We have seen that computing an arbitrary $\mathcal{K}_{m}$ has the great advantage that evolution can be computed in polynomial time. However, its non-determinism is a disadvantage. Clearly, we can avoid nondeterminism if we impose a linear order on the assertions over the signature of $\mathcal{K}$, and let BoldExpansion and BoldContraction choose them in this order. The question how to define such an order is again application-dependent and is out of the scope of our work.

A natural question that requires further investigation is whether there exist preferences as to which $\mathcal{K}_{m}$ to use for constructing the result of evolution such that they are generic enough and can be implemented without breaking tractability.
One may also wonder whether it is possible to efficiently compute a $\mathcal{K}_{m}$ with maximal cardinality. Recall that our algorithm is only guaranteed to compute a $\mathcal{K}_{m}$ that is maximal w.r.t. set inclusion. Unfortunately, it turns out that under this requirement computation is hard, even if $\mathcal{K}$ is either a TBox or an $\mathrm{ABox}$ and $\mathcal{N}$ is a TBox.

Theorem 5.7. Given $D L$-Lite $\mathcal{F}_{\mathcal{R}} K B s \mathcal{K}$ and $\mathcal{N}$ and a subset $\mathcal{K}_{0} \subseteq \mathcal{K}$ such that $\mathcal{K}_{0} \cup \mathcal{N}$ is coherent, deciding whether $\mathcal{K}_{0}$ has maximal cardinality among the elements of $\mathcal{M}_{e}(\mathcal{K}, \mathcal{N})$ is $N P$-complete. Moreover, NP-hardness already holds for DL-

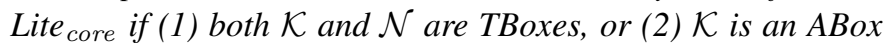
and $\mathcal{N}$ is a TBox.

Proof. This problem is equivalent to the problem of deciding whether there exists a subset $\mathcal{K}_{1}$ of $\mathcal{K}$ such that $\mathcal{K}_{1} \cup \mathcal{N}$ is coherent and $\left|\mathcal{K}_{1}\right| \geq\left|\mathcal{K}_{0}\right|+1$. We prove now that this latter problem is NP-complete. Indeed, the membership in NP is obvious: guess a subset $\mathcal{K}_{1}$ of $\mathcal{K}$ of size greater than $\left|\mathcal{K}_{0}\right|$ and check whether $\mathcal{K}_{1} \cup \mathcal{N}$ is coherent, which can be done in polynomial time. We show hardness by a reduction of the Independent Set Problem for graphs to the problem of evolution of a $D L$-Lite FR $_{\mathcal{R}}$ KB under bold semantics. Given a graph $G=(V, E)$, a subset $V^{\prime}$ of $V$ is called independent, if for any pair $u$ and $v$ in $V^{\prime}$ the edge $(u, v)$ is not in $E$. Deciding whether for a given integer $m \leq|V|$ an independent set of size $m$ or more exists is known to be NP-complete.

To prove the statement for Case 1, we use the following reduction. The TBox $\mathcal{T}$ consists of the assertions $S \sqsubseteq A_{i}$ for each $v_{i} \in \mathcal{V}$, and the new information $\mathcal{N}$ consists of the assertions $A_{i} \sqsubseteq \neg A_{j}$ for each $\left(v_{i}, v_{j}\right) \in E$. Clearly, a subset $\mathcal{T}_{1}=\left\{S \sqsubseteq A_{k} \mid k \in\left\{i_{1}, \ldots, i_{m}\right\}\right\}$ of $\mathcal{T}$ has the property that $\mathcal{T}_{1} \cup \mathcal{N}$ is coherent if and only if $\left\{v_{i_{1}}, \ldots, v_{i_{m}}\right\}$ is an independent set.

To prove the statement for Case 2, we use the following reduction. The ABox $\mathcal{A}$ consists of the membership assertions $A_{i}(b)$ for each $v_{i} \in V$, and the new information $\mathcal{N}$ is as in the previous case. Clearly, a subset $\mathcal{A}_{1}=\left\{A_{i_{1}}(b), \ldots, A_{i_{m}}(b)\right\}$ of $\mathcal{A}$ is such that $\mathcal{A}_{1} \cup \mathcal{N}$ is coherent if and only if $\left\{v_{i_{1}}, \ldots, v_{i_{m}}\right\}$ is an independent set.

In the next section we will see that nondeterminism is not present in ABox evolution, where the TBox is protected, and that there is always a single maximal compatible ABox.

\section{Formula-based Approaches to ABox Evolution}

In this section we study ABox evolution under formula-based approaches. First, observe that the classical approaches, $C P$ and WIDTIO, can be easily adapted to ABox evolution by requiring additionally that $\mathcal{T}$ is a part of $\mathcal{K}_{m}$. Note that this additional requirement does not contradict the general definition of $\mathcal{K}_{m}$. Indeed,

- In the case of expansion, since in the case of ABox evolution we assume that $\mathcal{T} \cup \mathcal{N}$ is coherent, the requirement that $\mathcal{T} \subseteq \mathcal{K}_{m}$ does not contradict that $\mathcal{K}_{m} \cup \mathcal{N}$ is coherent. 
- In the case of contraction, since a $D L$-Lite $_{\mathcal{F} \mathcal{R}}$ TBox does not entail any ABox assertion, the requirement that $\mathcal{T} \subseteq$ $\mathcal{K}_{m}$ does not contradict that $\mathcal{K}_{m} \forall \neq \alpha$, for each $\alpha \in \mathcal{N}$.

This requirement, however, brings a surprising result: it makes a maximal subset $\mathcal{K}_{m}$ unique.

Proposition 6.1. Let $\mathcal{K}=\mathcal{T} \cup \mathcal{A}$ be a $D$ L-Lite ${ }_{\mathcal{F} \mathcal{R}} K B$. Then

- If $\mathcal{K} \models \alpha_{1}$, where $\alpha_{1}$ is a DL-Lite $\mathcal{F}_{\mathcal{R}}$ membership assertion, then there exists $\alpha_{2} \in \mathcal{A}$ such that $\mathcal{T} \cup\left\{\alpha_{2}\right\} \models \alpha_{1}$.

- If $\mathcal{K}$ is unsatisfiable, then there exist $\alpha_{1}, \alpha_{2} \in \mathcal{A}$ such that $\mathcal{T} \cup\left\{\alpha_{1}, \alpha_{2}\right\}$ is unsatisfiable.

Proof. The proposition directly follows from the results in [15].

Proposition 6.1 immediately gives us the following lemma.

Lemma 6.2. Let $\mathcal{K}$ be a DL-Lite ${ }_{\mathcal{F} R} K B$ with TBox $\mathcal{T}$ and $\mathcal{N}$ a $D L$-Lite $\mathcal{F}_{\mathcal{R}}$ ABox. Then there exists exactly one element $\mathcal{K}_{m}$ in $\mathcal{M}_{e}(\mathcal{K}, \mathcal{N})$ (resp., in $\mathcal{M}_{c}(\mathcal{K}, \mathcal{N})$ ) such that $\mathcal{T} \subseteq \mathcal{K}_{m}$.

Proof. Suppose $\mathcal{K}=\mathcal{T} \cup \mathcal{A}$. Then $\mathcal{K}_{m}$ is obtained by dropping from $\mathcal{K}$, for each $\beta \in \mathcal{N}$, all ABox assertions $\alpha \in \mathcal{A}$ such that $\mathcal{T} \cup\{\beta, \alpha\}$ is unsatisfiable.

The straightforward consequence of this property is that the classical formula-based approaches, $C P$ and WIDTIO, and the proposed bold semantics coincide. Also observe that ABox evolution under bold semantics becomes deterministic, so we will use the binary operators $o_{b}$ and $\bullet_{b}$ to designate ABox expansion and contraction, respectively, under bold semantics.

Corollary 6.3. Let $\mathcal{K}=\mathcal{T} \cup \mathcal{N}$ be a $D L$-Lite ${ }_{\mathcal{F} \mathcal{R}} K B$ and $\mathcal{N}$ a DL-Lite $\mathcal{F}_{\mathcal{R}}$ ABox. Then, assuming that $\mathcal{T} \cup \mathcal{N}$ is coherent, ABox expansion (resp., ABox contraction) under CP, WIDTIO, and bold semantics coincide.

Next we study whether bold semantics satisfies the evolution postulates in the case of ABox evolution.

Proposition 6.4. For ABox evolution of DL-Lite $\mathcal{F}_{\mathcal{R}}$ KBs under bold semantics the following holds:

- ABox expansion satisfies $\boldsymbol{E 1 - E 5}$;

- ABox contraction satisfies C1-C3 and C5, but not C4.

Proof. The claim follows from Proposition 5.5 and the observation that in the case when $\mathcal{M}_{e}(\mathcal{K}, \mathcal{N})\left(\operatorname{resp} ., \mathcal{M}_{c}(\mathcal{K}, \mathcal{N})\right)$ is a singleton, EiB implies Ei (resp., C5B implies C5). The fact that contraction does not satisfy $\mathbf{C 4}$ can be shown as in Proposition 5.5

In principle, BoldExpansion and BoldContraction can be used to compute ABox evolution under bold semantics (and also $C P$ and WIDTIO) with the only change in Line 1 that we set $\mathcal{K}^{\prime}:=\mathcal{T} \cup \mathcal{N}$ in BoldExpansion and $\mathcal{K}^{\prime}:=\mathcal{T}$ in BoldContraction. Regardless of the order in which the algorithms select the assertions, they will always return the same result. A drawback of the algorithms is that they respectively perform a coherence and entailment check during each loop iteration. We exhibit now new algorithms FastExpansion and

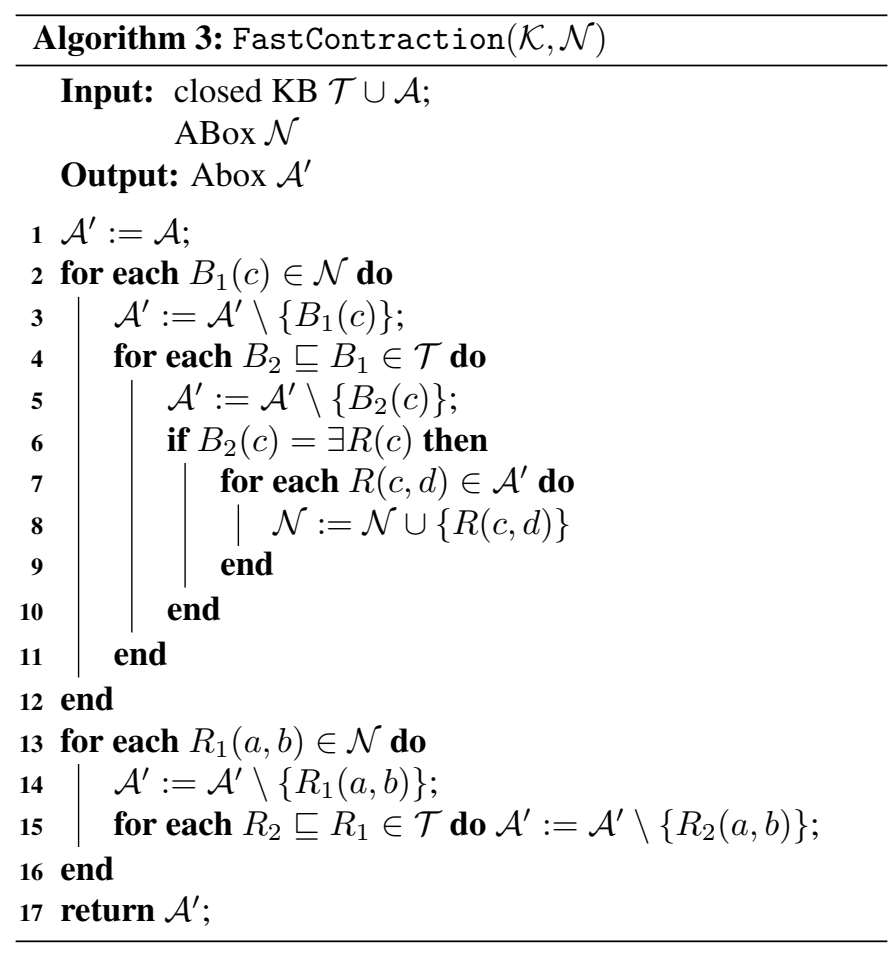

FastContraction that do not perform those checks; instead, they perform checks at the syntax level.

We start with the algorithm FastContraction. The algorithm (see Algorithm 3) works as follows: it takes as input a closed $D L$-Lite $_{\mathcal{F} \mathcal{R}} \mathrm{KB} \mathcal{T} \cup \mathcal{A}$ and a set of $D L$-Lite FR $_{\mathcal{R}}$ ABox assertions $\mathcal{N}$, and returns as output an ABox $\mathcal{A}^{\prime}$ such that (i) $\mathcal{A}^{\prime} \subseteq \mathcal{A}$ and (ii) $\mathcal{T} \cup \mathcal{A}^{\prime} \not \models \alpha$ for each $\alpha \in \mathcal{N}$. Now we show the correctness of the algorithm.

Theorem 6.5. The algorithm FastContraction computes an ABox contraction under bold semantics, that is, $(T \cup \mathcal{A}) \bullet_{b} \mathcal{N}=$ $\mathcal{T} \cup$ FastContraction $(T \cup \mathcal{A}, \mathcal{N})$, and runs in polynomial time.

Proof. The proof is based on the proof of Lemma6.2. Let $\mathcal{A}^{\prime}=$ FastContraction $(T \cup \mathcal{A}, \mathcal{N})$ and $\mathcal{K}^{\prime}=\mathcal{T} \cup \mathcal{A}^{\prime}$. We show that $\mathcal{K}^{\prime} \in \mathcal{M}_{c}(\mathcal{K}, \mathcal{N})$. First, we show that $\mathcal{K}^{\prime} \not=\alpha$ for each $\alpha \in \mathcal{N}$. Indeed, assume that this is not the case and there is an $\alpha \in \mathcal{N}$ such that $\mathcal{K}^{\prime} \models \alpha$. We know that there exists an inclusion assertion $\varphi \in \mathcal{T}$ and a membership assertion $\beta \in \mathcal{A}^{\prime}$ such that $\{\varphi, \beta\} \models \alpha$. We have five possible cases:

- $\alpha=\beta$. In this case we have that $\beta$ was removed from $\mathcal{A}^{\prime}$ at Line 3 during the corresponding loop iteration.

- $\alpha$ is of the form $B_{1}(c), \beta$ is of the form $B_{2}(c)$, and $\varphi$ is of the form $B_{2} \sqsubseteq B_{1}$. But then $\beta$ was removed from $\mathcal{A}^{\prime}$ at Line 5.

- $\alpha$ is of the form $\exists R(c), \beta$ is of the form $R(c, d)$, and $\varphi$ does not matter. In this case we have that $\beta$ was added to $\mathcal{N}$ at Line 8 and removed from $\mathcal{A}^{\prime}$ at Line 14 .

- $\alpha$ is of the form $\exists R_{1}(c), \beta$ is of the form $R_{2}(c, d)$, and $\varphi$ is of the form $R_{2} \sqsubseteq R_{1}$. In this case we have that $R_{1}(c, d) \in$ 


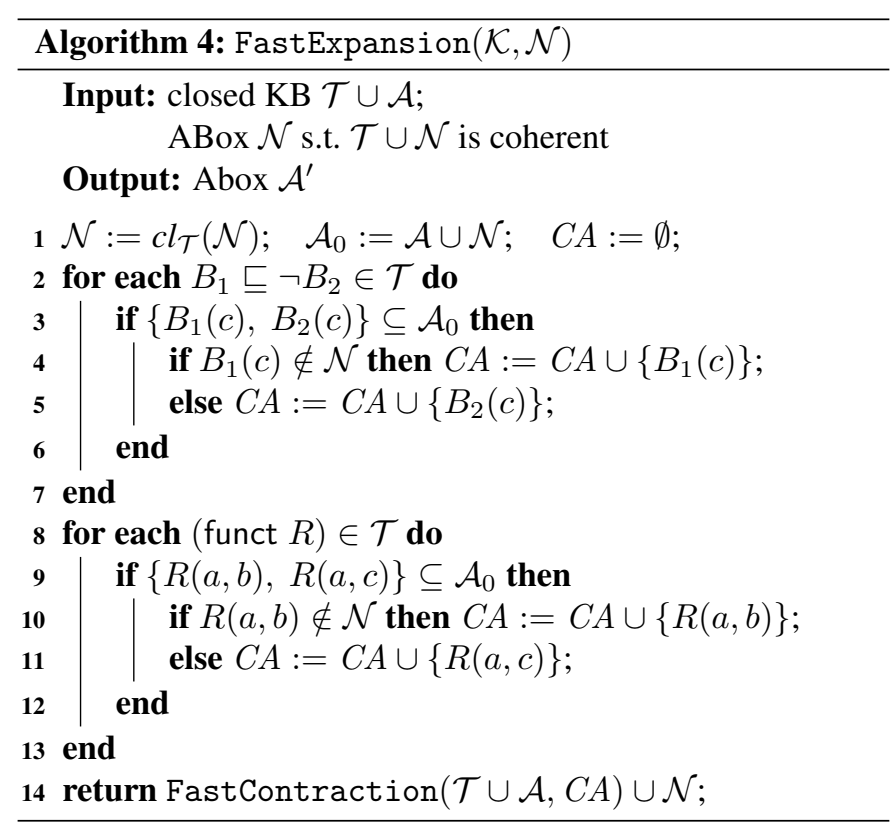

$\mathcal{A}$ and it was added to $\mathcal{N}$ at line 8 , and then $\beta$ was removed from $\mathcal{A}^{\prime}$ at Line 15 .

- $\alpha$ is of the form $R_{1}(a, b), \beta$ is of the form $R_{2}(a, b)$, and $\varphi$ is of the form $R_{2} \sqsubseteq R_{1}$. But then $\beta$ was removed from $\mathcal{A}^{\prime}$ at Line 15 .

In any case we have a contradiction.

The maximality of $\mathcal{K}^{\prime}$ follows straightforwardly from the following observation: if a membership assertion $\beta$ is from $\mathcal{K} \backslash \mathcal{K}^{\prime}$, then it was removed from $\mathcal{A}^{\prime}$ at Line $3,5,14$, or 15 . Then clearly, $\mathcal{K}^{\prime} \cup\{\beta\} \models \alpha$ for some $\alpha \in \mathcal{N}$, which shows the maximality of $\mathcal{K}^{\prime}$ and concludes the proof.

Now we turn to FastExpansion (see Algorithm 4). First, the algorithm detects the assertions in $\mathcal{A}$ that conflict with the new information $\mathcal{N}$ and stores them in $C A$. Then it resolves these conflicts using FastContraction as a subroutine. Finally, the algorithm returns the conflict-free part of $\mathcal{A}$ together with $\mathcal{N}$.

Theorem 6.6. The algorithm FastExpansion computes an ABox expansion under bold semantics, that is, $(\mathcal{T} \cup \mathcal{A}) \circ_{b} \mathcal{N}=$ $\mathcal{T} \cup$ FastExpansion $(\mathcal{T} \cup \mathcal{A}, \mathcal{N})$, and runs in polynomial time.

Proof. Let $\mathcal{K}=\mathcal{T} \cup \mathcal{A}, \mathcal{A}^{\prime}=$ FastContraction $(\mathcal{K}, C A)$, where $C A$ is as built by the algorithm from Lines $1-13$, and $\mathcal{K}^{\prime}=\mathcal{T} \cup \mathcal{A}^{\prime}$. We show that $\mathcal{K}^{\prime} \in \mathcal{M}_{e}(\mathcal{K}, \mathcal{N})$. First, we show that $\mathcal{K}^{\prime} \cup \mathcal{N}$ is consistent. Indeed, assume that this is not the case. We know [15] that there exists a TBox assertion $\varphi$ of the form $B_{1} \sqsubseteq \neg B_{2}$ or (funct $R$ ) and a pair of membership assertions $\alpha \in \mathcal{N}$ and $\beta \in \mathcal{A}^{\prime}$ such that $\{\varphi, \alpha, \beta\}$ is inconsistent. We have two possible cases:

- $\varphi$ is of the form $B_{1} \sqsubseteq \neg B_{2}, \alpha$ is of the form $B_{1}(c)$ (resp., $B_{2}(c)$ ), and $\beta$ is of the form $B_{2}(c)$ (resp., $B_{1}(c)$ ). But then we have that $\beta$ was added to $C A$ at Line 5 (resp., Line 4) and removed from $\mathcal{A}$ by FastContraction.
- $\varphi$ is of the form (funct $R$ ), $\alpha$ is of the form $R(a, b)$, and $\beta$ is of the form $R(a, c)$. But then we have that $\beta$ was added to $C A$ at Line 10 or 11 and removed from $\mathcal{A}$ by FastContraction.

In any case, we have a contradiction.

The maximality of $\mathcal{K}^{\prime}$ follows straightforwardly from the following observation: if a membership assertion $\beta$ is from $\mathcal{K} \backslash \mathcal{K}^{\prime}$, then it was removed from $\mathcal{A}$ by FastContraction and thus, added to $C A$ at Line $4,5,10$, or 11 . Then clearly, $\mathcal{K}^{\prime} \cup\{\beta\} \cup \mathcal{N}$ is inconsistent, which shows the maximality of $\mathcal{K}^{\prime}$ and concludes the proof.

Note that both Algorithm 3 and Algorithm 4 expect a closed $\mathrm{KB} \mathcal{K}$ as input. The algorithms can be optimised so as to deal with non-closed KBs. However, this kind of optimisation is outside the scope of our work.

\section{Related Work}

We provide an overview of related work, concentrating mostly on propositional logic and on Description Logics.

\subsection{Evolution in Propositional Logic KBs}

One of the first systematic studies of knowledge evolution that set the foundations of the area has been conducted by Alchourrón, Gärdenfors, and Makinson [24]. This work is commonly accepted as the most influential in the field of knowledge evolution and belief revision. The reason is that it proposed, on philosophical grounds, a set of rationality postulates that the operations of revision (adding information) and contraction (deleting information) must satisfy. Note that it used the term revision instead of expansion, which is used in this paper, and, in fact, that term is more commonly found in the literature. The postulates were well accepted by the research community and nowadays they are known as AGM postulates, named after the three authors who proposed them.

Dalal [30] introduced the principle of irrelevance of syntax, which states that the KB resulting from evolution should not depend on the syntax (or representation) of the old KB and the new information. A number of evolution approaches that meet the AGM postulates as well as Dalal's principle were proposed in the literature; the most well-known are by Fagin, Ullman, and Vardi [32], Borgida [28], Weber [33], Ginsberg [34], Dalal [30], Winslett [35], Satoh [29], and Forbus [27].

Winslett [9, 36] proposed the classification of evolution semantics into model-based semantics and formula-based semantics, which is the distinction that we have adopted in this paper. The operators from [32, 34] fall into the latter category, while the rest of the works cited above fall into the former category.

Katsuno and Mendelzon [37] gave a model-theoretic characterisation of model-based revision semantics that satisfied the AGM postulates. Keller and Winslett [38] introduced a taxonomy of knowledge evolution that is orthogonal to the one in [9]. They distinguished two types of adding information in the context of extended relational databases: changerecording updates and knowledge-adding updates. Later on 
Katsuno and Mendelzon [7] extended this work to the evolution of $\mathrm{KBs}$, referring to change-recording updates as updates and to knowledge-adding updates as revision. Intuitively, an update brings the KB up to date when the real world changes. The statement "John got divorced and now he is a priest" is an example of an update. Instead, revision is used when one obtains some new information about a static world. For example, we may try to diagnose a disease and we want to incorporate into the $\mathrm{KB}$ the result of successive tests. Incorporation of these tests is revision of the old knowledge. Both update and revision have applications where one is more suitable than the other. Moreover, Katsuno and Mendelzon showed that the AGM postulates and the model-theoretic characterisation of [37] are applicable to revision only. To fill the gap, they provided postulates and a model-theoretic characterisation for updates [7]. Their model-theoretic characterisation became prevalent in the $\mathrm{KB}$ evolution and belief revision literature.

\subsection{Evolution of Description Logic KBs}

Much less is known about the evolution of Description Logic knowledge bases than about the evolution of propositional logic, and the study of the topic is rather fragmentary.

Kang and Lau [39] discussed the feasibility of using the concept of belief revision as a basis for DL ontology revision. Flouris, Plexousakis, and Antoniou [40, 41] generalised the AGM postulates in order to apply the rationalities behind the AGM postulates to a wider class of logics, and determined the necessary and sufficient conditions for a logic to support the AGM postulates. However, none of [39, 40, 41] considered the explicit construction of a revision operator. Qi, Liu, and Bell [42] reformulated the AGM postulates for revision and adapted them to deal with disjunctive KBs expressed in the well-known DL $\mathcal{A L C}$.

Later, Qi, et al. [43] proposed a general revision operator to deal with incoherence. However, this operator is not finegrained, in the sense that it removes from a KB a whole TBox axiom by an incision function as soon as it affects the KB's coherency.

Haase and Stojanovic [44] proposed a formula-based approach for ontologies in OWL-Lite (which is a DL that is much more expressive than DL-Lite), where the removal of inconsistencies between the old and the new knowledge is strongly syntax-dependent. Notice instead that our formula-based semantics are syntax independent.

Liu et al. [14] considered several standard DLs of the $\mathcal{A L C}$ family [18], and studied the problem of ABox updates with empty TBoxes, in the case where the new information consists of atomic (possibly negated) ABox statements. They showed that these DLs are not closed even under simple updates. However, when the DLs are extended with nominals and the "@” constructor of hybrid logic [45], or, equivalently, admit nominal and Boolean ABoxes, then updates can be expressed. They also provided algorithms to compute updated ABoxes for several expressive DLs and studied the size of the resulting ABoxes. They showed that in general such ABoxes are exponential in the size of the update and the role-nesting depth of the original ABox, but that the exponential blowup can be avoided by considering so-called projective updates. They also consider conditional updates and how they can be applied to the problem of reasoning about actions.

The latter problem is also the motivation for Ahmetaj et al. [46], who study the evolution of extensional data under integrity constraints formulated in very expressive DLs of the $\mathcal{A L C}$ family, and in $D L$-Lite. The updates are finite sequences of conditional insertions and deletions, where complex DL formulas are used to select the (pairs of) nodes for which (node or arc) labels are added or deleted. The updates are finite sequences of conditional insertions and deletions, in which complex DL formulas are used to select the (pairs of) individuals to insert or remove from atomic concepts/roles. The paper studies the complexity of verifying when a sequence of update operations preserves the integrity constraints, by using a form of regression that reduces the problem to satisfiability checks over the initial KB. [47] extends the results on verification to the case where the DL may contain constructs for path-like navigation over the data.

Qi and Du [12] considered a model-based revision operator for DL terminologies (i.e., KBs with empty ABoxes) by adapting Dalal's operator. They showed that subsumption check-

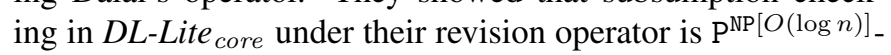
complete and provided a polynomial time algorithm to compute the result of revision for a specific class of input KBs. Observe that with the same argument as the one we used in the proof of Theorem 4.5. one can show that the expansion operator ${ }^{\prime} M^{\prime}$ of [12] (and its stratified extension $\circ_{S}$ ), is not expressible in $D L$-Lite $_{\mathcal{F} \mathcal{R}}$. This operator is a variant of $\mathbb{G}_{\#}^{s}$, where in Equation (1) one considers only models $\mathcal{J} \in \operatorname{Mod}(\mathcal{N})$ that satisfy $A^{\mathcal{J}} \neq \emptyset$ for every $A$ occurring in $\mathcal{K} \cup \mathcal{N}$. The modification does not affect the inexpressibility, which can again be shown using Example 4.3. We also note that $\circ_{M^{\prime}}$ was developed for $\mathrm{KB}$ expansion with empty ABoxes and the inexpressibility comes from the non-empty ABox.

De Giacomo et al. [13] considered ABox-update and erasure for the DL $D L$-Lite F. The $_{\text {. Th }}$ considered Winslett's approach (originally proposed for relational theories [9]) and showed that $D L-$ Lite $_{\mathcal{F}}$ is not closed under ABox-level update and erasure. The results in Section 4 extend these results in the following directions: (i) we showed new inexpressibility results for many other operators, including the operator from [13], and (ii) we considered both expansion and contraction at both $\mathrm{KB}$ and ABox level.

Wang, Wang, and Topor [48] introduced a new semantics for DL KBs and adapted to it the MBA. In contrast to classical model-based semantics, where evolution is based on manipulation with first-order interpretations, their approach is based on manipulation of so-called features, which are similar to models. In contrast to models, features are always of finite size and any DL KB has only finitely many features. They applied featurebased semantics to DL-Lite $e_{\text {bool }}^{\mathcal{N}}$ [23], and it turned out that the approach suffers from the same issues as classical model-based semantics. For example, DLs are not closed under these semantics even for simple evolution settings. Due to these problems, they addressed approximation of evolution semantics, but it turned out to be intractable. We conjecture that their seman- 
tics fits into our framework or Section 4 after a suitable extension, but our work does not extend their results. However, observe that the inexpressibility results of [48] reaffirm our arguments in Section 4, where we argued that model-based approaches suffer from intrinsic expressibility problems.

Lenzerini and Savo [49] considered the "when in doubt throw it out" (WIDTIO) approach for the case of DL-Lite ${ }_{A, i d}$ and presented a polynomial time algorithm for computing the evolution of KBs at the instance-level. Qi et al. [50] considered the problem of computing a maximal sound approximation of $D L$ Lite $_{\mathcal{F} \mathcal{R}} \mathrm{KB}$ expansions for two model-based operators. De Giacomo et al. [51] took a different approach to instance-level formula-based update of DL-Lite KBs: given an update specification, they rewrite it into a set of addition and deletion instructions over the ABox, which can be characterized as the result of a first-order query. This was proved by showing that every update can be reformulated into a Datalog program that generates the set of insertion and deletion instructions to change the ABox while preserving its consistency w.r.t. the TBox. De Giacomo et al. [52] looked at practical aspects of ontology update management in the context of ontology-based data access, where ontologies are 'connected' to relational data sources via declarative mappings [53]. In this scenario they study changes or evolution that affect ontologies and the source data and show how changes can be computed via non-recursive Datalog.

\subsection{Consistent Query Answering Over Inconsistent KBs}

Knowledge evolution is closely related to consistent query answering over inconsistent KBs, see e.g. [54, 54, 55, 56, 57], where the goal is, given a query $Q$ and an inconsistent $\mathrm{KB} \mathcal{K}$, to retrieve 'meaningful' answers for $Q$ over $\mathcal{K}]^{8}$ This problem has originally been introduced in the context of databases [58] and then adapted to KBs. Meaningful answers are typically defined using the notion of repairs: a $\mathrm{KB} \mathcal{K}^{\prime}$ is a repair of $\mathcal{K}$ if it is consistent and can be obtained by 'modifying' $\mathcal{K}$, e.g., by taking a (set-inclusion maximal) consistent subset of $\mathcal{K}$ (or its deductive closure). Then, semantics of $Q$ over $\mathcal{K}$ is defined as the intersection of ans $\left(Q, \mathcal{K}^{\prime}\right)$ over all repairs $\mathcal{K}^{\prime}$ of $\mathcal{K}$ that are optimal w.r.t. some criterion. Thus, query answering over inconsistent $\mathrm{KBs}$ is related to formula-based approaches to evolution, and in particular to WIDTIO, while to the best of our knowledge no work considers MBAs to KB repair. Observe that results analogous to our coNP-completeness of WIDTIO (see Theorem 5.3, which we first reported in [17], have been shown in the context of consistent query answering after our work has been published, e.g., in [54, 55, 57].

\subsection{Justification and Pinpointing}

Approaches to knowledge evolution that are often used in practice, in particular for TBox evolution, are essentially syntactic [44, 59, 60]. Many of them are based on justification or pinpointing: a minimal subset of the ontology that entails a

\footnotetext{
${ }^{8}$ Note that since $\mathcal{K}$ is inconsistent it holds that $\mathcal{K} \models Q(\vec{c})$ for every tuple $\vec{c}$ of constants with the $\operatorname{arity}(Q)$ and thus every tuple of constants of the appropriate arity is an answer to $Q$ over $\mathcal{K}$.
}

given consequence [61, 62, 63, 64, 65]. For example, to contract $\mathcal{K}$ with an assertion $\varphi$ entailed by $\mathcal{K}$, it suffices to compute all justifications for $\varphi$ in $\mathcal{K}$, find a minimal subset $\mathcal{K}_{1}$ of $\mathcal{K}$ with at least one assertion from each justification, and take $\mathcal{K}^{\prime}=\mathcal{K} \backslash \mathcal{K}_{1}$ as the result of evolution. This complies with a 'syntactical' notion of minimal change: retracting $\varphi$ requires to delete a minimal set of assertions from $\mathcal{K}$ and hence the structure of $\mathcal{K}$ is maximally preserved. Moreover, such $\mathcal{K}^{\prime}$ always exists even for expressive DLs, and practical algorithms to compute it have been implemented in ontology development platforms [65, 66]. By removing $\mathcal{K}_{1}$ from $\mathcal{K}$, however, we may inadvertently retract consequences of $\mathcal{K}$ other than $\varphi$, which are 'intended'. Identifying and recovering such intended consequences is an important issue. Evolution approaches considered in our work are logic-based rather than syntactic. Cuenca Grau et al. [67] present a framework to bridge the gap between logic-based and syntactic evolution approaches. In particular they propose a new principle of minimal change that has two dimensions: a structural one $\left(\mathcal{K}^{\prime}\right.$ should not change much the structure of $\mathcal{K}$ ) and a deductive one (that corresponds to the one we have for formula based evolution). Their work is focused on the DLs of the $\mathcal{E} \mathcal{L}$ family and does not consider model based evolution, which is crucial in our study. Moreover their evaluation algorithm for what they call finite preservation languages ( $D L$-Lite is included in this case) corresponds to a combination of our BoldExpansion and BoldContraction algorithms.

\subsection{Diagnosis and Debugging}

In diagnosis and debugging [62, 63, 68] the goal is to find the $\mathrm{KB}$ assertions that cause inconsistency. This is relevant since, e.g., a formula-based expansion of a KB with new knowledge can lead to its inconsistency and thus debugging techniques can help in finding what causes this inconsistency. There are attempts to relate these areas and $\mathrm{KB}$ evolution, e.g., Ribeiro and Wassermann [69] show how debugging services can be linked to belief revision. However, further investigation is required to gain a deeper understanding of the relation.

\section{Conclusions and Future Work}

In this paper we have studied evolution of $D L$-Lite KBs, taking into account both expansion and contraction. We have considered two main families of approaches: model-based ones and formula-based ones. We have singled out and investigated a three-dimensional space of model-based approaches, and have proven that most of them are not appropriate for DL-Lite, due to their counterintuitive behavior and the inexpressibility of evolution results. Thus, we have examined formula-based approaches, have shown that the classical ones are again inappropriate for DL-Lite, and have proposed a novel semantics called bold. We have shown that this semantics can be computed in polynomial time, but the result is, in general, non-deterministic. Then, we have studied ABox evolution under bold semantics and have shown that in this case the result is unique. We have developed polynomial time algorithms for DL-Lite KB expansion and contraction under this semantics, and alternative optimized variants of the algorithms for ABox evolution. 
The first important conclusion from our work is that modelbased approaches are intrinsically problematic for KB evolution, even in the case of such a lightweight DL as DL-Lite. Indeed, recall that DL-Lite is not closed under evolution for any of the model-based semantics and thus these semantics are impractical. As a consequence, one has either to search for conceptually different semantics that rely on other principles of 'composing' the output set of models constituting the evolution result, or one has to develop natural restrictions on how model-based approaches can 'compose' this set. An alternative approach would be to develop approximation techniques that allow one to efficiently capture evolution results.

A second important conclusion is that classical formulabased approaches are too heavyweight from the computational point of view and thus their practicality is questionable. On the other hand, the most conceptually simple model-based semantics such as bold semantics can potentially lead to practical evolution algorithms. However, their practicality requires further empirical evaluation. Finally, we have discussed that the classical evolution postulates that were originally developed for propositional theories are not directly applicable to the case of first-order knowledge since they are blind to some fundamental properties of such knowledge, such as coherency. We have shown how to adapt such postulates to the richer setting considered here, and have analyzed whether the various model-based and formula-based semantics satisfy the revised postulates.

We believe that our work opens new avenues for research in the area of knowledge evolution, which is an important part of knowledge engineering, since it shows how to lift approaches to knowledge evolution from the propositional to the first-order case. Moreover, we have presented techniques that allow one to prove inexpressibility of model-based evolution, and coNPhardness of formula-based evolution. We believe that these techniques can be relevant to knowledge management tasks beyond evolution.

We see several important directions for future work. First, the problem of expressibility in DL-Lite is still open for various model-based evolution semantics (see Table 1). These settings are all for ABox expansion and contraction under global modelbased semantics. An important research direction is to apply in practice the ideas we developed and, in particular, to implement an ontology evolution system. The system can be based on formula-based approaches and implement Algorithms 1-4 that we proposed. Such system could also be based on approximations of model-based semantics, which are out of the scope of this paper, see, e.g., [13, 70]. Then, it would be interesting to conduct an empirical evaluation for various semantics, in order to establish which semantics give more intuitive results from the users' point of view, and which ABox evolution approaches are more scalable. A further direction to investigate is to identify the minimum extensions of $D L$-Lite that would allow it to capture the results of model-based evolution for DL-Lite KBs. For this, one can draw inspiration from the work in [14], already discussed in Section 7 Also, it is still unknown what are minimal DLs that are closed under local model-based evolution, and in general that are well tailored towards model-based approaches. Finally, we believe that it is important to develop knowledge evolution techniques where the user has a much better control over the evolution process. For this, one can draw inspiration from previous work, e.g., from [67], where the authors proposed techniques to control what syntactic structures of a given $\mathrm{KB}$ cannot be changed by the evolution process, or from [71], where the authors proposed to combine knowledge evolution with models of trust, i.e., the new knowledge in their approach is only partially trusted (note that this scenario inherits the inexpressibility issues of MBAs).

Acknowledgements. This work was partially funded by the EPSRC projects $\mathrm{MaSI}^{3}$, DBOnto, $\mathrm{ED}^{3}$, by the UNIBZ projects PARCIS and TaDaQua, and by the "European Region Tyrol-South Tyrol-Trentino" (EGTC) under the first call for basic research projects within the Euregio Interregional Project Network IPN12 "Knowledge-Aware Operational Support" (KAOS).

\section{References}

[1] A. Borgida, R. J. Brachman, Conceptual modeling with description logics, in: Baader et al. [18], Ch. 10, pp. 349-372.

[2] D. L. Martin, M. H. Burstein, D. V. McDermott, S. A. McIlraith, M. Paolucci, K. P. Sycara, D. L. McGuinness, E. Sirin, N. Srinivasan, Bringing semantics to web services with OWL-S, World Wide Web J. 3 (2007) 243-277. doi:10.1007/s11280-007-0033-x

[3] D. Berardi, D. Calvanese, G. De Giacomo, R. Hull, M. Mecella, Automatic composition of transition-based semantic web services with messaging, in: Proc. of the 31st Int. Conf. on Very Large Data Bases (VLDB), 2005, pp. 613-624.

[4] S. A. Mcilraith, T. C. Son, H. Zeng, Semantic web services, IEEE Intelligent Systems 16 (2) (2001) 46-53.

[5] U. Kuter, E. Sirin, B. Parsia, D. S. Nau, J. A. Hendler, Information gathering during planning for web service composition, J. of Web Semantics 3 (2-3) (2005) 183-205.

[6] G. Flouris, D. Manakanatas, H. Kondylakis, D. Plexousakis, G. Antoniou, Ontology change: Classification and survey, Knowledge Engineering Review 23 (2) (2008) 117-152.

[7] H. Katsuno, A. Mendelzon, On the difference between updating a knowledge base and revising it, in: Proc. of the 2nd Int. Conf. on the Principles of Knowledge Representation and Reasoning (KR), 1991, pp. 387-394.

[8] S. Abiteboul, G. Grahne, Update semantics for incomplete databases, in: Proc. of the 11th Int. Conf. on Very Large Data Bases (VLDB), 1985, pp. $1-12$.

[9] M. Winslett, Updating Logical Databases, Cambridge University Press, 1990.

[10] T. Eiter, G. Gottlob, On the complexity of propositional knowledge base revision, updates and counterfactuals, Artificial Intelligence 57 (1992) 227-270.

[11] G. Flouris, On belief change in ontology evolution, AI Communications-The Eur. J. on Artificial Intelligence 19 (4).

[12] G. Qi, J. Du, Model-based revision operators for terminologies in description logics, in: Proc. of the 21 st Int. Joint Conf. on Artificial Intelligence (IJCAI), 2009, pp. 891-897.

[13] G. De Giacomo, M. Lenzerini, A. Poggi, R. Rosati, On instance-level update and erasure in description logic ontologies, J. of Logic and Computation 19 (5) (2009) 745-770.

[14] H. Liu, C. Lutz, M. Milicic, F. Wolter, Foundations of instance level updates in expressive description logics, Artificial Intelligence 175 (18) (2011) 2170-2197.

[15] D. Calvanese, G. De Giacomo, D. Lembo, M. Lenzerini, R. Rosati, Tractable reasoning and efficient query answering in description logics: The DL-Lite family, J. of Automated Reasoning 39 (3) (2007) 385-429.

[16] F. Baader, S. Brandt, C. Lutz, Pushing the $\mathcal{E} \mathcal{L}$ envelope, in: Proc. of the 19th Int. Joint Conf. on Artificial Intelligence (IJCAI), 2005, pp. 364-369. 
[17] D. Calvanese, E. Kharlamov, W. Nutt, D. Zheleznyakov, Evolution of DL-Lite knowledge bases, in: Proc. of the 9th Int. Semantic Web Conf. (ISWC), Vol. 6496 of Lecture Notes in Computer Science, Springer, 2010, pp. 112-128. doi:10.1007/978-3-642-17746-0\_8

[18] F. Baader, D. Calvanese, D. McGuinness, D. Nardi, P. F. Patel-Schneider (Eds.), The Description Logic Handbook: Theory, Implementation and Applications, Cambridge University Press, 2003.

[19] A. Poggi, D. Lembo, D. Calvanese, G. De Giacomo, M. Lenzerini, R. Rosati, Linking data to ontologies, J. on Data Semantics 10 (2008) 133-173. doi:10.1007/978-3-540-77688-8\55

[20] B. Motik, B. Cuenca Grau, I. Horrocks, Z. Wu, A. Fokoue, C. Lutz, OWL 2 Web Ontology Language profiles (second edition), W3C Recommendation, World Wide Web Consortium, available at http://www . w3.org/TR/owl2-profiles/(Dec. 2012).

[21] B. Cuenca Grau, I. Horrocks, B. Motik, B. Parsia, P. Patel-Schneider, U. Sattler, OWL 2: The next step for OWL, J. of Web Semantics 6 (4) (2008) 309-322.

[22] J. Bao, et al., OWL 2 Web Ontology Language document overview (second edition), W3C Recommendation, World Wide Web Consortium, available at http://www.w3.org/TR/owl2-overview/(Dec. 2012).

[23] A. Artale, D. Calvanese, R. Kontchakov, M. Zakharyaschev, The DL-Lite family and relations, J. of Artificial Intelligence Research 36 (2009) 1-69.

[24] C. E. Alchourrón, P. Gärdenfors, D. Makinson, On the logic of theory change: Partial meet contraction and revision functions, J. of Symbolic Logic 50 (2) (1985) 510-530.

[25] M. L. Ginsberg, D. E. Smith, Reasoning about action I: A possible worlds approach, Tech. Rep. KSL-86-65, Knowledge Systems, AI Laboratory (1987).

[26] D. Zheleznyakov, Logic-based evolution of knowledge bases: The case of lightweight DLs, Ph.D. thesis, Free University of Bozen-Bolzano, Bolzano, Italy (May 2013).

[27] K. D. Forbus, Introducing actions into qualitative simulation, in: Proc. of the 11th Int. Joint Conf. on Artificial Intelligence (IJCAI), 1989, pp. 1273-1278

[28] A. Borgida, Language features for flexible handling of exceptions in information systems, ACM Trans. on Database Systems 10 (4) (1985) 565603.

[29] K. Satoh, Nonmonotonic reasoning by minimal belief revision, in: Proc. of the Int. Conf. on 5th Generation Computer Systems (FGCS), 1988, pp. 455-462.

[30] M. Dalal, Investigations into a theory of knowledge base revision, in: Proc. of the AAAI Conf. on Artificial Intelligence (AAAI), 1988, pp. 475-479.

[31] D. Calvanese, E. Kharlamov, W. Nutt, A proof theory for DL-Lite, in: Proc. of the 20th Int. Workshop on Description Logics (DL), Vol. 250 of CEUR Workshop Proceedings, http://ceur-ws.org/. 2007, pp. 235242.

[32] R. Fagin, J. D. Ullman, M. Y. Vardi, On the semantics of updates in databases, in: Proc. of the 2nd ACM Symp. on Principles of Database Systems (PODS), 1983, pp. 352-365.

[33] A. Weber, Updating propositional formulas, in: Proc. of the 1st Int. Conf. on Expert Database Systems (EDBS), 1986, pp. 487-500.

[34] M. L. Ginsberg, Counterfactuals, Artificial Intelligence 30 (1) (1986) 3579.

[35] M. Winslett, Reasoning about action using a possible models approach, in: Proc. of the 7th Nat. Conf. on Artificial Intelligence (AAAI), 1988, pp. 89-93.

[36] M. Winslett, A framework for comparison of update semantics, in: Proc of the 7th ACM Symp. on Principles of Database Systems (PODS), 1988, pp. 315-324.

[37] H. Katsuno, A. O. Mendelzon, A unified view of propositional knowledge base updates, in: Proc. of the 11th Int. Joint Conf. on Artificial Intelligence (IJCAI), Morgan Kaufmann, 1989, pp. 1413-1419.

[38] A. M. Keller, M. Winslett, On the use of an extended relational model to handle changing incomplete information, IEEE Trans. on Software Engineering 11 (7) (1985) 620-633.

[39] S. H. Kang, S. K. Lau, Ontology revision using the concept of belief revision, in: Proc. of the 8th Int. Conf. on Knowledge-Based Intelligent Information \& Engineering Systems (KES), 2004, pp. 261-267.

[40] G. Flouris, D. Plexousakis, G. Antoniou, Generalizing the AGM postulates: Preliminary results and applications, in: Proc. of the 10th Int
Workshop on Non-Monotonic Reasoning (NMR), 2004, pp. 171-179.

[41] G. Flouris, D. Plexousakis, G. Antoniou, On applying the AGM theory to DLs and OWL, in: Proc. of the 4th Int. Semantic Web Conf. (ISWC), Vol. 3729 of Lecture Notes in Computer Science, Springer, 2005, pp. 216-231.

[42] G. Qi, W. Liu, D. A. Bell, Knowledge base revision in description logics, in: Proc. of the 10th Eur. Conf. on Logics in Artificial Intelligence (JELIA), 2006, pp. 386-398.

[43] G. Qi, P. Haase, Z. Huang, Q. Ji, J. Z. Pan, J. Voelker, A kernel revision operator for terminologies - Algorithms and evaluation, in: Proc. of the 7th Int. Semantic Web Conf. (ISWC), Vol. 5318 of Lecture Notes in Computer Science, Springer, 2008, pp. 419-434. doi:10.1007/ 978-3-540-88564-1\_27

[44] P. Haase, L. Stojanovic, Consistent evolution of OWL ontologies, in: Proc. of the Extended Semantic Web Conf. (ESWC), Vol. 3532 of Lecture Notes in Computer Science, Springer, 2005, pp. 182-197.

[45] C. Areces, M. de Rijke, From description to hybrid logics, and back, in: Advances in Modal Logics Volume 3, 2000, pp. 17-36.

[46] S. Ahmetaj, D. Calvanese, M. Ortiz, M. Simkus, Managing change in graph-structured data using description logics, ACM Trans. on Computational Logic 18 (4) (2017) 27:1-27:35. doi:10.1145/3143803

[47] D. Calvanese, M. Ortiz, M. Simkus, Verification of evolving graphstructured data under expressive path constraints, in: Proc. of the 19th Int. Conf. on Database Theory (ICDT), Vol. 48 of Leibniz Int. Proc. in Informatics (LIPIcs), Schloss Dagstuhl-Leibniz-Zentrum für Informatik, Dagstuhl, Germany, 2016, pp. 15:1-15:19.

[48] Z. Wang, K. Wang, R. W. Topor, A new approach to knowledge base revision in DL-Lite, in: Proc. of the 24th AAAI Conf. on Artificial Intelligence (AAAI), 2010, pp. 369-374.

[49] M. Lenzerini, D. F. Savo, On the evolution of the instance level of DL-Lite knowledge bases, in: Proc. of the 24th Int. Workshop on Description Logics (DL), Vol. 745 of CEUR Workshop Proceedings, http: //ceur-ws.org/ 2011.

[50] G. Qi, Z. Wang, K. Wang, X. Fu, Z. Zhuang, Approximating model-based ABox revision in DL-Lite: Theory and practice, in: Proc. of the 29th AAAI Conf. on Artificial Intelligence (AAAI), 2015, pp. 254-260.

[51] G. De Giacomo, X. Oriol, R. Rosati, D. F. Savo, Updating DL-Lite ontologies through first-order queries, in: Proc. of the 15th Int. Semantic Web Conf. (ISWC), Vol. 9981 of Lecture Notes in Computer Science, 2016, pp. 167-183. doi:10.1007/978-3-319-46523-4\_11

[52] G. De Giacomo, D. Lembo, X. Oriol, D. F. Savo, E. Teniente, Practical update management in ontology-based data access, in: Proc. of the 16th Int. Semantic Web Conf. (ISWC), Vol. 10588 of Lecture Notes in Computer Science, Springer, 2017, pp. 225-242. doi:10.1007/ 978-3-319-68288-4\_14

[53] G. Xiao, D. Calvanese, R. Kontchakov, D. Lembo, A. Poggi, R. Rosati, M. Zakharyaschev, Ontology-based data access: A survey, in: Proc. of the 27th Int. Joint Conf. on Artificial Intelligence (IJCAI), AAAI Press, 2018.

[54] M. Bienvenu, On the complexity of consistent query answering in the presence of simple ontologies, in: Proc. of the 26th AAAI Conf. on Artificial Intelligence (AAAI), 2012, pp. 705-711.

[55] D. Lembo, M. Lenzerini, R. Rosati, M. Ruzzi, D. F. Savo, Inconsistencytolerant query answering in ontology-based data access, J. of Web Semantics 33 (2015) 3-29.

[56] M. Bienvenu, R. Rosati, Tractable approximations of consistent query answering for robust ontology-based data access, in: Proc. of the $23 \mathrm{rd}$ Int. Joint Conf. on Artificial Intelligence (IJCAI), 2013, pp. 775-781.

[57] M. Bienvenu, C. Bourgaux, F. Goasdoué, Query-driven repairing of inconsistent DL-Lite knowledge bases, in: Proc. of the 25th Int. Joint Conf. on Artificial Intelligence (IJCAI), 2016, pp. 957-964.

[58] M. Arenas, L. E. Bertossi, J. Chomicki, Consistent query answers in inconsistent databases, in: Proc. of the 18th ACM Symp. on Principles of Database Systems (PODS), 1999, pp. 68-79.

[59] A. Kalyanpur, B. Parsia, E. Sirin, B. Cuenca Grau, Repairing unsatisfiable concepts in OWL ontologies, in: Proc. of the 3rd European Semantic Web Conf. (ESWC), Vol. 4011 of Lecture Notes in Computer Science, Springer, 2006, pp. 170-184. doi:10.1007/11762256\_15

[60] E. Jiménez-Ruiz, B. Cuenca Grau, I. Horrocks, R. B. Llavori, Supporting concurrent ontology development: Framework, algorithms and tool, Data and Knowledge Engineering 70 (1) (2011) 146-164. 
[61] R. Peñaloza, B. Sertkaya, On the complexity of axiom pinpointing in the EL family of description logics, in: Proc. of the 12th Int. Conf. on the Principles of Knowledge Representation and Reasoning (KR), 2010.

[62] A. Kalyanpur, B. Parsia, E. Sirin, J. A. Hendler, Debugging unsatisfiable classes in OWL ontologies, J. of Web Semantics 3 (4) (2005) 268-293.

[63] S. Schlobach, R. Cornet, Non-standard reasoning services for the debugging of description logic terminologies, in: Proc. of the 18th Int. Joint Conf. on Artificial Intelligence (IJCAI), 2003, pp. 355-362.

[64] S. Schlobach, Z. Huang, R. Cornet, F. van Harmelen, Debugging incoherent terminologies, J. of Automated Reasoning 39 (3) (2007) 317-349.

[65] A. Kalyanpur, B. Parsia, M. Horridge, E. Sirin, Finding all justifications of OWL DL entailments, in: Proc. of the 6th Int. Semantic Web Conf. (ISWC), Vol. 4825 of Lecture Notes in Computer Science, Springer, 2007, pp. 267-280. doi:10.1007/978-3-540-76298-0\_20

[66] B. Suntisrivaraporn, G. Qi, Q. Ji, P. Haase, A modularization-based approach to finding all justifications for OWL DL entailments, in: Proc. of the 3rd Asian Semantic Web Conference (ASWC), Vol. 5367 of Lecture Notes in Computer Science, Springer, 2008, pp. 1-15. doi:10.1007/ 978-3-540-89704-0\_1

[67] B. Cuenca Grau, E. Jiménez-Ruiz, E. Kharlamov, D. Zheleznyakov, Ontology evolution under semantic constraints, in: Proc. of the 13th Int. Conf. on the Principles of Knowledge Representation and Reasoning (KR), AAAI Press, 2012, pp. 137-147.

[68] M. Horridge, B. Parsia, U. Sattler, Explaining inconsistencies in OWL ontologies in: Proc. of the 3rd Int. Conf. on Scalable Uncertainty Management (SUM), Vol. 5785 of Lecture Notes in Computer Science, Springer, 2009, pp. 124-137. doi:10.1007/978-3-642-04388-8\_11 URL https://doi.org/10.1007/978-3-642-04388-8_11

[69] M. M. Ribeiro, R. Wassermann, Base revision for ontology debugging, J. of Logic and Computation 19 (5) (2009) 721-743.

[70] E. Kharlamov, D. Zheleznyakov, D. Calvanese, Capturing model-based ontology evolution at the instance level: The case of DL-Lite, J. of Computer and System Sciences 79 (6) (2013) 835-872. doi:10.1016/j. jcss.2013.01.0065

[71] D. Zheleznyakov, E. Kharlamov, I. Horrocks, Trust-sensitive evolution of DL-Lite knowledge bases, in: Proc. of the 31st AAAI Conf. on Artificial Intelligence (AAAI), 2017, pp. 1266-1273. 